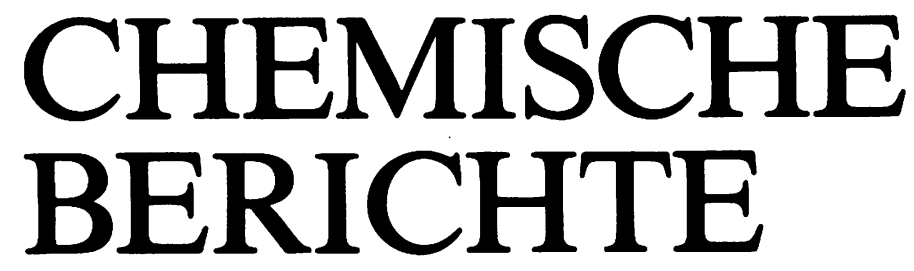

GEGRÜNDET 1868

115. JAHRGANG

HERAUSGEGEBEN IM AUFTRAG DER

GESELLSCHAFT DEUTSCHER CHEMIKER

VON

K. HAFNER - W. KIRMSE - H. MUSSO - H. NÖTH • J. SAUER - E. WINTERFELDT

UNTER MITWIRKUNG VON

H. A. BRUNE - W. LÜTTKE - G. SPITELLER

REDAKTION: H. ZAHN

mit H. SCHILL, J. STREHLOW und A. WIELAND 
Schaumann Ernst, Bäuch Hans-Günther, Sieveking Stefan und Adiwidjaja Gunadi: Cycloadditionsreaktionen von Heterocumulenen, XXV: Cycloaddukte und Umlagerungsprodukte aus der Umsetzung von Isothiocyanaten mit Keten-acetalen . ........

Neidlein Richard und Zeiner Hartmut: Synthesen einiger ungesättigter Carbonsäuren des 1,6-Methano[10]annulens sowie des überbrückten Dicyanpseudophenalen-fulvens . .

Hellmann Goetz, Hellmann Siegried, Beckhaus Hans-Dieter und Rüchardt Christoph: Thermolabile Kohlenwasserstoffe, XVI: Thermische Stabilität, Spannungsenthalpie und Struktur symmetrisch tetrasubstituierter Ethane $\ldots \ldots \ldots \ldots \ldots \ldots \ldots \ldots$

Heuschmann Manfred und Quast Helmut: Dreigliedrige Heterocyclen, 14: $\alpha$-Halogenierung von tertiären Phosphanoxiden $\ldots \ldots \ldots \ldots \ldots \ldots \ldots \ldots \ldots \ldots \ldots \ldots$

Auchter Gerhard und Hanack Michael: Vinylkationen, 38: Synthese und Solvolyse 3-sub-

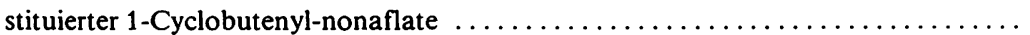

Cetinkaya Bekir, Binger Paul und Krüger Carl: Metallacycloalkane, V: Darstellung eines Rhodacycloheptans durch oxidative Kopplung von 3,3-Dimethylcyclopropen an

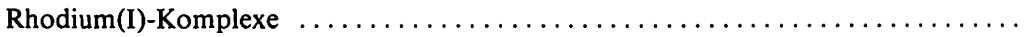

Welt Günther, Wolf Elisabeth, Fischer Peter und Föhlisch Baldur: Unterschiedliche [4 + 2]-Cycloaddukte aus 7,7-Difluor- und 7,7-Dialkoxy-1,3,5-cycloheptatrienen mit

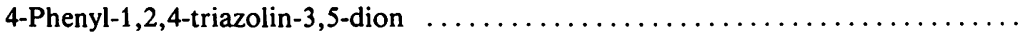

Rumiński Jan K. und Przewoska Krystyna D.: Synthese und Reaktionsfähigkeit von 2-Aroylbenzoesäuren, II: 2-(4-Hydroxy-3-isopropylbenzoyl)benzoesäure . . . . . .

Weidenbruch Manfred, Flott Hermann, Fleischhauer Jörg und Schleker Wolfgang: Siliciumverbindungen mit starken intramolekularen sterischen Wechselwirkungen, 13: Rotationshinderungen in Tri-tert-butylsilanen . . . . . . . . . . . . . . . .

Zander Maximilian: Über die Umsetzung von Carbazolylkalium mit 9-Bromanthracen

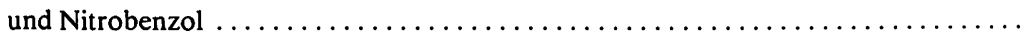




\section{PHYSICAL CHEMISTRY}

Hellmann Goetz, Hellmann Siegried, Beckhaus Hans-Dieter, and Rüchardt Christoph: Thermolabile Hydrocarbons, XVI: Thermal Stability, Strain Enthalpy, and Structure of Sym. Tetrasubstituted Ethanes $\ldots \ldots \ldots \ldots \ldots \ldots \ldots \ldots \ldots \ldots \ldots \ldots \ldots$

\section{INORGANIC CHEMISTRY}

Richter Felix and Vahrenkamp Heinrich: Chiral SFeCoM Clusters: Synthesis, Side

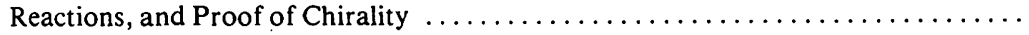

Richter Felix and Vahrenkamp Heinrich: Chiral SFeCoM Clusters: Enantiomer Separation and Determination of the Absolute Configuration $\ldots \ldots \ldots \ldots \ldots \ldots$

Keçeci Ahmet, Rehder Dieter, Roose Wolfgang, and Talay Ridvan: Phosphane-bridged Dinuclear Complexes Containing Carbonyl- $\eta^{5}$-cyclopentadienyl Transition Metal

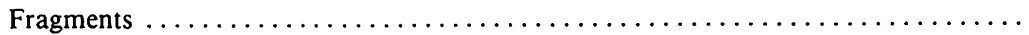

Maringgele Walter: Reaction of Metal and Metalloid Compounds with Polyfunctional Molecules, XXXVI: New Syntheses of Open-Chain and Cyclic $\mathrm{N}$-Borylureas and

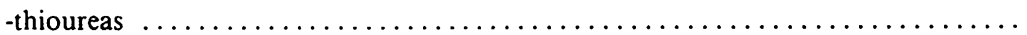

Schmidbaur Hubert, Deschler Ulrich, and Milewski-Mahrla Beatrix: Ylide Complexes of Alkali and Alkaline Earth Metals, V: Pyridine-substituted Phosphonium-bis-methylide Anions as Chelating Ligands for Sodium, Potassium, and Barium. Crystal Structure of a Binuclear Potassium Complex ........................

Sohn Dieter and Sundermeyer Wolfgang: Reaction of Pentafluoro-2-aza-1-propene with

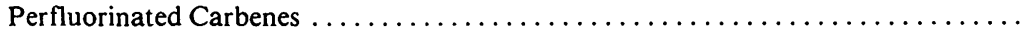

Köster Hajo and Weiss Erwin: Metal Alkyl and Aryl Compounds, XXVIII: Preparation and Crystal Structure of Allyllithium- $N, N, N^{\prime}, N^{\prime}$-Tetramethylethylenediamine,

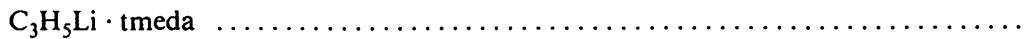

Weidenbruch Manfred, Flott Hermann, Fleischhauer Jörg, and Schleker Wolfgang: Silicon Compounds with Strong Intramolecular Steric Interactions, 13: Restricted Rotations in Tri-tert-butylsilanes

\section{ORGANIC CHEMISTRY}

Leininger Hartmut, Kemmer Petra, Beck Karin, and Christl Manfred: 7-Thiatetracyclo$\left[4 \cdot 1.0 .0^{2,4} \cdot 0^{3,5}\right]$ heptane (Benzvalene Sulfide) - Synthesis and Reactions . . . . . . .

Maringgele Walter: Reaction of Metal and Metalloid Compounds with Polyfunctional Molecules, XXXVI: New Syntheses of Open-Chain and Cyclic $\mathrm{N}$-Borylureas and

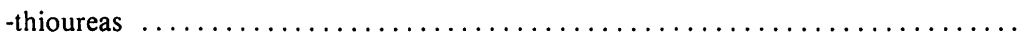

Erker Gerhard, Wicher Joachim, Engel Klaus, and Krüger Carl: (s-trans- $\eta^{4}$-Diene)zirconocene Complexes

Erker Gerhard, Engel Klaus, Krüger Carl, and Chiang An-Pei: Reactivity and Structure of (s-cis-1,3-Diene)zirconocene Complexes

Roedig Alfred and Ritschel Werner: Reactions of 3,4,4-Trichloro-3-butenamides with Nucleophiles, I: Dimers and Alcohol Adducts of 1,1-Dichloroallenecarboxamides .. 
Schaumann Ernst, Bäuch Hans-Günther, Sieveking Stefan, and Adiwidjaja Gunadi: Cycloaddition Reactions of Heterocumulenes, XXV: Cycloadducts and Rearrangement Products from the Reaction of Isothiocyanates with Ketene Acetals ..........

Neidlein Richard and Zeiner Hartmut: Syntheses of Some Unsaturated Carboxylic Acids of 1,6-Methano[10]annulene and the Bridged Dicyanopseudophenalene-fulvene ....

Hellmann Goetz, Hellmann Siegried, Beckhaus Hans-Dieter, and Rüchardt Christoph: Thermolabile Hydrocarbons, XVI: Thermal Stability, Strain Enthalpy, and Structure of Sym. Tetrasubstituted Ethanes $\ldots \ldots \ldots \ldots \ldots \ldots \ldots \ldots \ldots \ldots \ldots$

Heuschmann Manfred and Quast Helmut: Three-membered Ring Heterocycles, 14: $\alpha$-Halogenation of Tertiary Phosphane Oxides $\ldots \ldots \ldots \ldots \ldots \ldots \ldots \ldots$

Auchter Gerhard and Hanack Michael: Vinyl Cations, 38: Synthesis and Solvolysis of

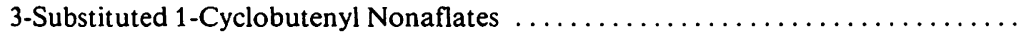

Cetinkaya Bekir, Binger Paul, and Krüger Carl: Metallacycloalkanes, V: Preparation of a Rhodacycloheptane by Oxidative Coupling of 3,3-Dimethylcyclopropene to

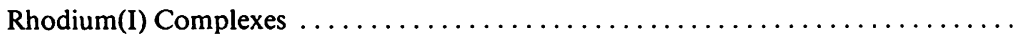

Welt Günther, Wolf Elisabeth, Fischer Peter, and Föhlisch Baldur: [4+2] Cycloadducts with Alternate Structure from 7,7-Difluoro- and 7,7-Dialkoxy-1,3,5-cycloheptatrienes and 4-Phenyl-1,2,4-triazoline-3,5-dione $\ldots \ldots \ldots \ldots \ldots \ldots \ldots \ldots$

Ruminski Jan K. and Przewoska Krystyna D.: Synthesis and Reactivity of 2-Aroylbenzoic Acids, II: 2-(4-Hydroxy-3-isopropylbenzoyl)benzoic Acid . . . . . . . . . . .

Weidenbruch Manfred, Flott Hermann, Fleischhauer Jörg, and Schleker Wolfgang: Silicon Compounds with Strong Intramolecular Steric Interactions, 13: Restricted Rotations in Tri-tert-butylsilanes

Zander Maximilian: Reaction of Carbazolylpotassium with 9-Bromoanthracene and Nitrobenzene 


\section{AUTORENREGISTER}

Adiwidjaja, G. s. Schaumann, E. . . . 3340 Auchter, G. und Hanack, M. . . . . . 3402

Bäuch, H.-G. s. Schaumann, E. . . . . 3340

Beck, K. s. Leininger, H. . . . . . . 3213

Beckhaus, H.-D. s. Hellmann, G. . . . 3364

Binger, P. s. Cetinkaya, B. ....... 3414

Cetinkaya, B., Binger, $P$. und

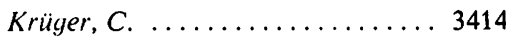

Chiang, A.-P. s. Erker, G. ........ 3311

Christl, M. s. Leininger, H. . . . . . 3213

Deschler, U. s. Schmidbaur, H. . . . . 3290

Engel, K. s. Erker, G. . . . . . 3 3300, 3311

Erker, G., Engel, K., Krüger, C. und

Chiang, A.-P. ........... 3311

-, Wicher, J., Engel, K. und

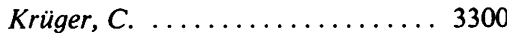

Fischer, P. s. Welt, G. . . . . . . . . 3427

Fleischhauer, J. s. Weidenbruch, M. .. 3444

Flott, $H$. s. Weidenbruch, $M$. ...... 3444

Föhlisch, B. s. Welt, G. . . . . . . . 3427

Hanack, M. s. Auchter, G. . . . . . . . 3402

Hellmann, G., Hellmann, S.,

Beckhaus, H.-D. und Rüchardt, C. 3364

Hellmann, S. s. Hellmann, G. . . . . . . 3364

Heuschmann, M. und Quast, H. ..... 3384

Keçeci, A., Rehder, D., Roose, $W$.

und Talay, $R . \ldots \ldots \ldots \ldots \ldots 257$

Kemmer, P. s. Leininger, H. . . . . . 3213

Köster, H. und Weiss, E. . . . . . . 3422

Krüger, C. s. Cetinkaya, B. . . . . . 3414

- s. Erker, G. . . . . . . . . . 3300, 3311

Leininger, H., Kemmer, P., Beck, $K$.

und Christl, $M$.

3213
Maringgele, $W . \ldots \ldots \ldots \ldots \ldots . \ldots . \ldots 3271$

Milewski-Mahrla, B. s. Schmidbaur, H. 3290

Neidlein, $R$. und Zeiner, H. . . . . . 3353

Przewoska, K. D. s. Rumiński, J. K. . . 3436

Quast, H. s. Heuschmann, M. . . . . 3384

Rehder, D. s. Keçeci, A. . . . . . . . 3257

Richter, F. und Vahrenkamp, H. . 3224, 3243

Ritschel, W. s. Roedig, A. . . . . . . 3324

Roedig, A. und Ritschel, W. . . . . . . 3324

Roose, W. s. Keçeci, A. . . . . . . . 3257

Rüchardt, C. s. Hellmann, G. . . . . . 3364

Rumiński, J. K. und Przewoska, K. D. . 3436

Schaumann, E., Bäuch, H.-G.,

Sieveking, S. und Adiwidjaja, G. . 3340

Schleker, W. s. Weidenbruch, M. ..... 3444

Schmidbaur, H., Deschler, $U$. und

Milewski-Mahrla, B. . . . . . . . 3290

Sieveking, S. s. Schaumann, E. . . . . 3340

Sohn, D. und Sundermeyer, W. ...... 3334

Sundermeyer, W. s. Sohn, D. . . . . . 3334

Talay, R. s. Keçeci, A. . . . . . . . 3257

Vahrenkamp, H. s. Richter, F. . . 3224, 3243

Weidenbruch, M., Flott, $H$.,

Fleischhauer, J. und Schleker, W. . 3444

Weiss, E. s. Köster, H. . . . . . . . 3422

Welt, G., Wolf, E., Fischer, P. und

Föhlisch, B. . . . . . . . . . . 3427

Wicher, J. s. Erker, G. . . . . . . . 3300

Wolf, E. s. Welt, G. .......... 3427

Zander, $M$. ............... 3449

Zeiner, $H$. s. Neidlein, $R$. . ...... 3353 


\title{
Additions- und Cycloadditionsreaktionen von Allenyl-Kationen mit acyclischen 1,3-Dienen
}

\author{
Herbert Mayr* und Herbert Klein \\ Institut für Organische Chemie der Universität Erlangen-Nürnberg, \\ Henkestr. 42, D-8520 Erlangen
}

Eingegangen am 1. März 1982

Die Zinkchlorid-Ether-katalysierte Umsetzung von Propargylchloriden 1 mit acyclischen Dienen 2 liefert bei $-78^{\circ} \mathrm{C}$ die linearen Additionsprodukte 3 bzw. 4, die unter Lewis-Säure-Katalyse bei höheren Temperaturen zu den cyclischen Vinylchloriden 5 umlagern. Nur solche Kombinationen aus 1 und 2 liefern dabei 1:1-Produkte, bei denen die Edukte 1 rascher dissoziieren als die Produkte. Andernfalls entstehen höhermolekulare Verbindungen wie z. B. das 2:1-Produkt 6. Die Reaktionsmechanismen der Additionsreaktionen werden diskutiert.

\section{Addition and Cycloaddition Reactions of Allenyl Cations with Acyclic 1,3-Dienes}

The zinc chloride-ether catalysed reactions of propargyl halides 1 with acyclic dienes 2 at $-78^{\circ} \mathrm{C}$ give linear addition products 3 and 4 . At elevated temperature allyl chlorides 3 and $\mathbf{4}$ rearrange to vinyl chlorides 5 with Lewis acid catalysis. Only those combinations of 1 and 2 give $1: 1$ products, in which educts 1 dissociate more rapidly than the reaction products. Otherwise high molecular weight compounds such as $2: 1$ product 6 are formed. The reaction mechanism of the addition reactions is discussed.

Cycloadditionsreaktionen von Allenyl-Kationen mit cyclischen 1,3-Dienen stellen einen einfachen Zugang zu zahlreichen bicyclischen Verbindungen dar 1,2). In dieser Arbeit berichten wir über die Bildung acyclischer und monocyclischer Additionsverbindungen durch Lewis-Säurekatalysierte Umsetzungen von Propargylchloriden mit acyclischen 1,3-Dienen 3).

\section{Reaktionsprodukte}

Die Zinkchlorid-Ether ${ }^{1}$-katalysierte Umsetzung der Propargylchloride 1 mit den Dienen 2 liefert üblicherweise bei $-78^{\circ} \mathrm{C}$ die Allylchloride 3 oder 4, die bei höheren Temperaturen unter Lewis-Säure-Katalyse zu den Cyclohexen-Derivaten $\mathbf{5}$ cyclisieren (Schema 1).

Wie Tab. 1 zeigt, ist es jedoch unter diesen Bedingungen nicht gelungen, das Dimethylpropargylchlorid 1a an 1,3-Butadien (2a) oder Isopren (2b) zu addieren. Während bei $-78^{\circ} \mathrm{C}$ keine Reaktion erfolgte, polymerisierte bei $0^{\circ} \mathrm{C}$ das jeweilige Dien.

Das Trimethylpropargylchlorid 1 b lieferte mit 2 a bei $-78^{\circ} \mathrm{C}$ ein $4: 1$-Gemisch der Allylchloride 3c und 4c. Daraus entsteht beim Behandeln mit Zinn(IV)-chlorid bei $-78^{\circ} \mathrm{C}$ reines $3 \mathrm{c}$, das bei $0^{\circ} \mathrm{C}$ unter Zinkchlorid-Ether-Katalyse langsam zu $(E)-5 \mathrm{c}$ isomerisiert. Während dieser Cyclisierung ist Luftausschluß erforderlich, da sonst, vermutlich über einen Radikalmechanismus, aromatische Nebenprodukte (Isopropyl- 
toluol?) in größeren Mengen entstehen. Aus 1b und Isopren (2b) erhält man bei $-78^{\circ} \mathrm{C}$ als einziges $1: 1$-Produkt das 1,4-Addukt 3d, das bei $0^{\circ} \mathrm{C}$ unter ZinkchloridKatalyse rasch zu $(E)-5 d$ umlagert. Bei der Umsetzung von $2 \mathbf{f}$ mit $\mathbf{1 b}$ konnten keine $1: 1$-Produkte isoliert werden.

Schema l<smiles>[R]C#CC([R])([R])CC([R1])=C([R7])C([R7])([R])Cl</smiles>

3<smiles>[R]C(Cl)=C1C2=C(Cl)C([R7])(CC([R])=C2[R])C1([R2])[R2]</smiles>

(E) $\mathbf{5}$<smiles>[R]C#CC([R])([R])Cl</smiles>

1<smiles>[R7]C(=C)C([R7])=C([R7])CC</smiles>

2<smiles>[R]C#CC([R])([R])CC([R7])(Cl)C([R7])=C([R])[R]</smiles>

4

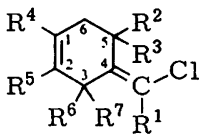

$(Z)-5$

\begin{tabular}{r|lll} 
& $\mathrm{R}^{1}$ & $\mathrm{R}^{2}$ & $\mathrm{R}^{3}$ \\
\hline $\mathbf{1 a}$ & $\mathrm{H}$ & $\mathrm{CH}_{3}$ & $\mathrm{CH}_{3}$ \\
$\mathbf{b}$ & $\mathrm{CH}_{3}$ & $\mathrm{CH}_{3}$ & $\mathrm{CH}_{3}$ \\
$\mathbf{c}$ & $\mathrm{Ph}$ & $\mathrm{CH}_{3}$ & $\mathrm{CH}_{3}$ \\
$\mathbf{d}$ & $\mathrm{Ph}$ & $\mathrm{Ph}$ & $\mathrm{H}$
\end{tabular}

\begin{tabular}{r|llll} 
& $\mathrm{R}^{4}$ & $\mathrm{R}^{5}$ & $\mathrm{R}^{6}$ & $\mathrm{R}^{7}$ \\
\hline $\mathbf{2 a}$ & $\mathrm{H}$ & $\mathrm{H}$ & $\mathrm{H}$ & $\mathrm{H}$ \\
$\mathbf{b}$ & $\mathrm{CH}_{3}$ & $\mathrm{H}$ & $\mathrm{H}$ & $\mathrm{H}$ \\
$\mathbf{c}$ & $\mathrm{H}$ & $\mathrm{H}$ & $\mathrm{CH}_{3}$ & $\mathrm{H}$ \\
$\mathbf{d}$ & $\mathrm{CH}_{3}$ & $\mathrm{CH}_{3}$ & $\mathrm{H}$ & $\mathrm{H}$ \\
$\mathbf{e}$ & $-\left[\mathrm{CH}_{2}\right]_{4}-$ & $\mathrm{H}$ & $\mathrm{H}$ \\
$\mathbf{f}$ & $\mathrm{H}$ & $\mathrm{H}$ & $\mathrm{CH}_{3}$ & $\mathrm{CH}_{3}$ \\
$\mathbf{g}$ & $\mathrm{CH}_{3}$ & $\mathrm{H}$ & $\mathrm{CH}_{3}$ & $\mathrm{H}$ \\
$\mathbf{h}$ & $\mathrm{CH}_{3}$ & $\mathrm{H}$ & $\mathrm{CH}_{3}$ & $\mathrm{CH}_{3}$
\end{tabular}

\begin{tabular}{r|lllllll} 
3-5 & $\mathrm{R}^{1}$ & $\mathrm{R}^{2}$ & $\mathrm{R}^{3}$ & $\mathrm{R}^{4}$ & $\mathrm{R}^{5}$ & $\mathrm{R}^{6}$ & $\mathrm{R}^{7}$ \\
\hline $\mathbf{a}$ & $\mathrm{H}$ & $\mathrm{CH}_{3}$ & $\mathrm{CH}_{3}$ & $\mathrm{H}$ & $\mathrm{H}$ & $\mathrm{H}$ & $\mathrm{H}$ \\
$\mathbf{b}$ & $\mathrm{H}$ & $\mathrm{CH}_{3}$ & $\mathrm{CH}_{3}$ & $\mathrm{CH}_{3}$ & $\mathrm{H}$ & $\mathrm{H}$ & $\mathrm{H}$ \\
$\mathbf{c}$ & $\mathrm{CH}_{3}$ & $\mathrm{CH}_{3}$ & $\mathrm{CH}_{3}$ & $\mathrm{H}$ & $\mathrm{H}$ & $\mathrm{H}$ & $\mathrm{H}$ \\
$\mathbf{d}$ & $\mathrm{CH}_{3}$ & $\mathrm{CH}_{3}$ & $\mathrm{CH}_{3}$ & $\mathrm{CH}_{3}$ & $\mathrm{H}$ & $\mathrm{H}$ & $\mathrm{H}$ \\
$\mathbf{e}$ & $\mathrm{CH}_{3}$ & $\mathrm{CH}_{3}$ & $\mathrm{CH}_{3}$ & $\mathrm{H}$ & $\mathrm{H}$ & $\mathrm{CH}_{3}$ & $\mathrm{CH}_{3}$ \\
$\mathbf{f}$ & $\mathrm{Ph}$ & $\mathrm{CH}_{3}$ & $\mathrm{CH}_{3}$ & $\mathrm{H}$ & $\mathrm{H}$ & $\mathrm{H}$ & $\mathrm{H}$ \\
$\mathbf{g}$ & $\mathrm{Ph}$ & $\mathrm{CH}_{3}$ & $\mathrm{CH}_{3}$ & $\mathrm{CH}_{3}$ & $\mathrm{H}$ & $\mathrm{H}$ & $\mathrm{H}$ \\
$\mathbf{h}$ & $\mathrm{Ph}$ & $\mathrm{CH}_{3}$ & $\mathrm{CH}_{3}$ & $\mathrm{H}$ & $\mathrm{H}$ & $\mathrm{CH}_{3}$ & $\mathrm{H}$ \\
$\mathbf{i}$ & $\mathrm{Ph}$ & $\mathrm{CH}_{3}$ & $\mathrm{CH}_{3}$ & $\mathrm{CH}_{3}$ & $\mathrm{CH}$ & $\mathrm{H}$ & $\mathrm{H}$ \\
$\mathbf{j}$ & $\mathrm{Ph}$ & $\mathrm{CH}_{3}$ & $\mathrm{CH}_{3}$ & $-\left[\mathrm{CH}_{2}\right]_{4}-$ & $\mathrm{H}$ & $\mathrm{H}$ \\
$\mathbf{k}$ & $\mathrm{Ph}$ & $\mathrm{CH}_{3}$ & $\mathrm{CH}_{3}$ & $\mathrm{H}$ & $\mathrm{H}$ & $\mathrm{CH}_{3}$ & $\mathrm{CH}$ \\
$\mathbf{l}$ & $\mathrm{Ph}$ & $\mathrm{CH}_{3}$ & $\mathrm{CH}_{3}$ & $\mathrm{CH}_{3}$ & $\mathrm{H}$ & $\mathrm{CH}_{3}$ & $\mathrm{H}$ \\
$\mathbf{m}$ & $\mathrm{Ph}$ & $\mathrm{CH}_{3}$ & $\mathrm{CH}_{3}$ & $\mathrm{CH}_{3}$ & $\mathrm{H}$ & $\mathrm{CH}_{3}$ & $\mathrm{CH}$ \\
$\mathbf{n}$ & $\mathrm{Ph}$ & $\mathrm{Ph}_{3}$ & $\mathrm{H}$ & $\mathrm{CH}_{3}$ & $\mathrm{H}$ & $\mathrm{H}$ & $\mathrm{H}$ \\
$\mathbf{o}$ & $\mathrm{Ph}$ & $\mathrm{Ph}$ & $\mathrm{H}$ & $\mathrm{H}$ & $\mathrm{H}$ & $\mathrm{CH}_{3}$ & $\mathrm{H}$ \\
$\mathbf{p}$ & $\mathrm{Ph}$ & $\mathrm{Ph}$ & $\mathrm{H}$ & $\mathrm{CH}_{3}$ & $\mathrm{H}$ & $\mathrm{CH}_{3}$ & $\mathrm{H}$
\end{tabular}

Das im Vergleich zu 1b reaktivere Propargylchlorid 1c liefert mit 1,3-Butadien (2a) bei $-78^{\circ} \mathrm{C}$ in Gegenwart von Zinkchlorid-Ether ein Gemisch der Isomeren $3 \mathbf{f}, \mathbf{4 f}$ und $(E)-5 f$. Behandelt man dieses Gemisch mit Lewis-Säure bei Raumtemperatur, lagert zunächst $\mathbf{4 f}$ in $(E)-\mathbf{5} \mathbf{f}$ um, und wesentlich langsamer folgt die Cyclisierung $\mathbf{3 f} \rightarrow(E)-\mathbf{5} \mathbf{f}$. Die alkylierten Diene $\mathbf{2 b}-\mathbf{h}$ reagieren mit $\mathbf{1 c}$ rascher als $\mathbf{2 a}$. Dabei erhält man mit Isopren (2b), Piperylen (2c) und 2,3-Dimethylbutadien (2d) bei $-78^{\circ} \mathrm{C}$ die Allylchloride $3 \mathbf{g}-\mathbf{i}$, begleitet durch unterschiedliche Mengen an $(E)-5 \mathrm{~g}-\mathbf{i}$. Bei $0^{\circ} \mathrm{C}$ lagern $3 \mathrm{~g}-\mathbf{i}$ in Gegenwart von Zinkchlorid-Ether vollständig in die Benzylidencyclohexene $(E)-5 \mathbf{g}-\mathbf{i}$ um. Bei der Umsetzung von 1c mit 1,2-Dimethylencyclohexan (2e), einem s-cis-fixierten 
Tab. 1. Zinkchlorid-katalysierte Umsetzungen von Propargylchloriden 1 mit 1,3-Dienen 2

\begin{tabular}{|c|c|c|c|c|c|c|c|}
\hline \multirow{2}{*}{\multicolumn{2}{|c|}{ Edukte }} & & \multirow{3}{*}{$\begin{array}{c}\begin{array}{c}\text { Ausb. } \\
(3-5, \%)\end{array} \\
0\end{array}$} & \multirow{3}{*}{\multicolumn{2}{|c|}{\begin{tabular}{cc}
\multicolumn{3}{c}{ Produkte } \\
Formel & $3: 4: 5$ \\
$a$ & -
\end{tabular}}} & \multirow{3}{*}{$\begin{array}{c}\begin{array}{c}\text { Cyclisierung } \\
\text { bei } T\left({ }^{\circ} \mathrm{C}\right)\end{array} \\
-\end{array}$} \\
\hline & & \multirow{2}{*}{$\frac{T\left({ }^{\circ} \mathrm{C}\right)}{-78 ; 0}$} & \multirow{2}{*}{$\frac{t(\mathrm{~h})}{\mathrm{b})}$} & & & & \\
\hline $1 \mathrm{a}$ & $2 a$ & & & & & & \\
\hline & 2b & $-78 ; 0$ & b) & 0 & b & - & - \\
\hline \multirow[t]{3}{*}{$1 \mathrm{~b}$} & $2 a$ & -78 & $120^{c)}$ & 36 & c & $80: 20: 0$ & 0 \\
\hline & 2b & -78 & 44 & 26 & d & $100: 0: 0$ & 0 \\
\hline & $2 f$ & -78 & 15 & 0 & e & - & - \\
\hline \multirow[t]{8}{*}{$1 \mathrm{c}$} & $2 a$ & -78 & $48^{c)}$ & 46 & f & $40: 40: 20$ & 25 \\
\hline & 2b & -78 & 4 & 67 & g & $91: 0: 9$ & 0 \\
\hline & $2 c$ & -78 & 4 & 70 & h & $96: 0: 4$ & -10 \\
\hline & 2d & -78 & 6 & 59 & $\mathbf{i}$ & $70: 0: 30$ & -10 \\
\hline & $2 \mathrm{e}$ & -40 & 2 & 44 & $\mathbf{j}$ & $0: 0: 100$ & $<-40$ \\
\hline & $2 f$ & -65 & 6 & $0^{\text {d) }}$ & k & - & - \\
\hline & $2 \mathrm{~g}$ & -78 & 6 & 0 & 1 & - & - \\
\hline & $2 \mathrm{~h}$ & -78 & 6 & 0 & m & - & - \\
\hline \multirow[t]{3}{*}{$1 \mathrm{~d}$} & $2 b$ & -78 & 6 & 78 & $\mathbf{n}$ & $100: 0: 0$ & 0 \\
\hline & $2 c$ & -78 & 18 & $89^{e)}$ & o & $67: 33: 0$ & -10 \\
\hline & $2 g$ & -78 & 6 & 57 & $\mathbf{p}$ & $0: 0: 100$ & $<-78$ \\
\hline
\end{tabular}

a) Zusammensetzung NMR-spektroskopisch bestimmt. - b) Keine Reaktion bei $-78^{\circ} \mathrm{C}$; Umlagerung von 1a zu 1-Chlor-3-methyl-1,2-butadien und Polymerisation des Diens bei $0^{\circ} \mathrm{C}$. - c) Zinkchlorid-Konzentration viermal so hoch wie bei den übrigen Versuchen. - d) $23 \%$ 6. - e) Ausbeute an isoliertem 5 nach Cyclisierung.

Dien, können bei $-40^{\circ} \mathrm{C}$ keine Allylchloride beobachtet werden, und man erhält direkt das Octahydronaphthalin $(E)-5 \mathbf{j}$. Die Dimethylbutadiene $2 \mathbf{f}$ und $\mathbf{g}$ sowie das Trimethylbutadien $\mathbf{2 h}$ liefern bei der Lewis-Säure-katalysierten Umsetzung mit 1c keine 1:1-Produkte. Während $\mathbf{2 g}$ und $\mathbf{h}$ ein kompliziertes Gemisch höhermolekularer Verbindungen ergaben, konnte bei der Umsetzung von 2 f mit 1c das 2:1-Produkt 6 mit $23 \%$ Ausbeute isoliert werden.

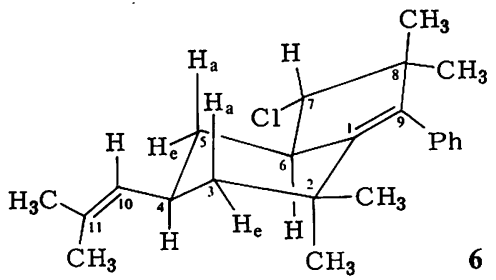

Ausschließlich lineare Additionsprodukte 3n bzw. ein Gemisch aus 30 und 40 erhält man bei der Zinkchlorid-Ether-katalysierten Umsetzung des Diphenylpropargylchlorids $1 \mathrm{~d}$ mit Isopren (2b) und Piperylen $(2 \mathrm{c})$ bei $-78^{\circ} \mathrm{C}$. In beiden Fällen erfolgt bei erhöhter Temperatur Cyclisierung zu den Vinylchloriden 5n und 5o. In Analogie zu den oben beschriebenen Cyclisierungen entsteht $(E)-\mathbf{5 n}$ isomerenrein, während $\mathbf{5 0}$ als $(E),(Z)$-Gemisch anfällt. Da in $(E),(Z)$-5o außerdem noch zwei Asymmetriezentren vorliegen, entsteht dieses Vinylchlorid als Gemisch von vier Diastereomeren. In gleicher 
Weise liefert $\mathbf{1 d}$ mit $\mathbf{2 g}$ vier diastereomere Cyclohexene $\mathbf{5 p}$, ohne daß hier lineare Zwischenstufen nachweisbar waren.

\section{Strukturaufklärung}

Die linearen Additionsprodukte 3 und 4 lassen sich durch ihre ${ }^{1} \mathrm{H}$-NMR-Spektren (Tab. 2) identifizieren, die größtenteils nach 1. Ordnung ausgewertet werden können. Als zusätzlicher Strukturbeweis kann ihre Umlagerung zu den Vinylchloriden $\mathbf{5}$ angesehen werden.

Tab. 2. ${ }^{1} \mathrm{H}-\mathrm{NMR}-\mathrm{Spektren} \mathrm{der} \mathrm{linearen} \mathrm{Additionsprodukte} 3$ in $\mathrm{CCl}_{4}[\delta$, Multiplizität, $J(\mathrm{~Hz})]$

\begin{tabular}{|c|c|c|c|c|c|c|c|c|c|}
\hline & $\mathrm{R}^{1}$ & $\mathrm{R}^{2}$ & $\mathrm{R}^{3}$ & $\mathrm{CH}_{2}$ & $\mathrm{R}^{4}$ & & $\mathrm{R}^{5}$ & $\mathrm{R}^{6}$ & $\mathrm{R}^{7}$ \\
\hline $3 c$ & $\begin{array}{l}1.77 \\
\mathrm{~s}\end{array}$ & $\begin{array}{l}1.13 \\
\mathrm{~s}\end{array}$ & $=\mathrm{R}^{2}$ & $\begin{array}{l}2.11 \\
\text { br. d, } 6\end{array}$ & $\begin{array}{l}5.7 \\
\mathrm{mc}\end{array}$ & & $\begin{array}{l}5.7 \\
\mathrm{mc}\end{array}$ & $=\mathrm{R}^{7}$ & $\begin{array}{l}4.02 \\
d, 6\end{array}$ \\
\hline 3d & $\begin{array}{l}1.71 \\
\mathrm{~s}\end{array}$ & $\begin{array}{l}1.12 \\
\mathrm{~s}\end{array}$ & $=\mathrm{R}^{2}$ & $\begin{array}{l}2.07 \\
\text { br. s }\end{array}$ & $\begin{array}{l}1.85 \\
\text { br. } \mathrm{s}\end{array}$ & & $\begin{array}{l}5.41 \\
\text { br. t, } 8\end{array}$ & $=\mathrm{R}^{7}$ & $\begin{array}{l}4.01 \\
d, 8\end{array}$ \\
\hline $3 f$ & $\begin{array}{l}7.16 \\
\mathrm{mc}\end{array}$ & $\begin{array}{l}1.25 \\
\mathrm{~s}\end{array}$ & $=\mathrm{R}^{2}$ & $\begin{array}{l}2.20 \\
d, 6\end{array}$ & $\begin{array}{l}5.74 \\
\mathrm{mc}\end{array}$ & & $\begin{array}{l}5.74 \\
\mathrm{mc}\end{array}$ & $=\mathrm{R}^{7}$ & $\begin{array}{l}3.97 \\
d, 6\end{array}$ \\
\hline $3 g$ & $\begin{array}{l}7.25 \\
\mathrm{mc}\end{array}$ & $\begin{array}{l}1.29 \\
\mathrm{~s}\end{array}$ & $=\mathrm{R}^{2}$ & $\begin{array}{l}2.23 \\
\text { br. s }\end{array}$ & $\begin{array}{l}1.92 \\
\text { br. } s\end{array}$ & & $\begin{array}{l}5.53 \\
\text { br. t, } 8\end{array}$ & $=\mathrm{R}^{7}$ & $\begin{array}{l}4.05 \\
d, 8\end{array}$ \\
\hline $3 \mathrm{~h}$ & $\begin{array}{l}7.23 \\
\mathrm{mc}\end{array}$ & $\begin{array}{l}1.26 \\
s\end{array}$ & $=\mathrm{R}^{2}$ & $\begin{array}{l}2.19 \\
d, 6\end{array}$ & $\begin{array}{l}5.36 \\
\mathrm{~m}\end{array}$ & - & 6.10 & $\begin{array}{l}1.57 \\
d, 7\end{array}$ & $\begin{array}{l}4.49 \\
\text { quint, } 7\end{array}$ \\
\hline$\left.(E, Z)-3 \mathbf{i}^{\mathbf{a}}\right)$ & $\begin{array}{l}7.23 \\
\mathrm{mc}\end{array}$ & $\begin{array}{l}1.30 \\
\mathrm{~s}\end{array}$ & $=\mathrm{R}^{2}$ & $\begin{array}{l}2.34 \\
\mathrm{~s}\end{array}$ & $\begin{array}{l}1.82 \\
\text { br. } \mathrm{s}\end{array}$ & & $\begin{array}{l}1.97 \\
2.02 \\
\mathrm{mc}\end{array}$ & $=\mathrm{R}^{7}$ & $\begin{array}{l}4.05 \\
\mathrm{~s}\end{array}$ \\
\hline $3 n$ & $\begin{array}{l}6.9- \\
\mathrm{m}\end{array}$ & 7.6 & $\underset{\left(t^{b)}\right.}{\approx 3.9}$ & $\begin{array}{l}2.47 \\
d, 8\end{array}$ & $\begin{array}{l}1.75 \\
\text { br. } \mathrm{s}\end{array}$ & & $\begin{array}{l}5.45 \\
\text { br. t, } 8\end{array}$ & $=\mathrm{R}^{7}$ & $\begin{array}{l}3.95 \\
d, 8\end{array}$ \\
\hline $3 o^{a)}$ & $\begin{array}{l}7.0- \\
\mathrm{m}\end{array}$ & 7.5 & $\begin{array}{l}\left.3.6-4.0^{c}\right) \\
\mathrm{m}\end{array}$ & $\begin{array}{l}2.3-2.6 \\
\mathrm{~m}\end{array}$ & $\begin{array}{l}5.25 \\
\mathrm{~m}\end{array}$ & - & 5.85 & $\begin{array}{l}1.47 \\
d, 7\end{array}$ & $\begin{array}{l}4.36 \\
\text { quint, } 7\end{array}$ \\
\hline
\end{tabular}

a) Identifizierung im Gemisch. - b) Teilweise verdeckt. - c) Wegen Überlagerung ist Tieffeldbegrenzung nicht eindeutig feststellbar.

Zur Identifizierung der cyclischen Produkte wurde $(E)-5 \mathrm{~g}$ mit überschüssigem Silbertrifluoracetat in Ether $90 \mathrm{~h}$ unter Rückfluß gekocht. Dabei erhielt man den Trifluoressigsäurevinylester 7, der sich im 'H-NMR-Spektrum von (E)-5g nur dadurch unterscheidet, daß das 3-H-Signal um $0.3 \mathrm{ppm}$ nach hohem Feld verschoben ist. Aminolyse von 7 liefert das Arylketon 8, das im IRSpektrum eine Carbonylbande bei $1670 \mathrm{~cm}^{-1}$ zeigt.<smiles>CC1=CCC(=C(Cl)Pc2ccccc2)C(C)(C)C1</smiles>

(E) $-5 \mathbf{g}$<smiles>CC1=CCC(=C(OC(=O)C(F)(F)F)C(C)(C)C)C(C)(C)C1</smiles>

7<smiles>CC1=CCC(C(=O)c2ccccc2)C(C)(C)C1</smiles>

8<smiles>[R4]C1=CCC(C)=C(Cl)C(C)(C)C1</smiles>

9

Die entsprechende Konstitution 5 der anderen cyclischen Verbindungen wird aus ihren ${ }^{1} \mathrm{H}$ NMR-Spektren gefolgert (Tab. 3). Das $\mathrm{C}=\mathrm{C}\left(\mathrm{CH}_{3}\right) \mathrm{Cl}$-Strukturelement in $\mathbf{5} \mathbf{c}$ und $\mathbf{d}$ ergibt sich aus der ${ }^{~} \mathrm{H}$-NMR-Absorption einer Methylgruppe bei $\delta=2.3$; in der alternativen Struktur 9 sollte die entsprechende Methylgruppe stärker abgeschirmt sein ${ }^{1,2}$ ).

Die Hoch feldverschiebung der Signale der Methylgruppen $\mathrm{R}^{2}$ und $\mathrm{R}^{3}$ in $\mathbf{5} \mathbf{f}-\mathbf{j}$ indiziert die $(E)$ Konfiguration dieser Vinylchloride, weil in diesen Isomeren die Methylgruppe im positiven Abschirmbereich des Phenylrests liegt. In $\mathbf{5} \mathbf{h}$, die als einzige dieser Verbindungen im zeitlichen Mittel 
nicht $C_{s}$-Symmetrie besitzt, ist eine Methylgruppe besonders stark abgeschirmt, während die andere im normalen Bereich absorbiert. Die ähnliche chemische Verschiebung von $3-H\left(=R^{6}, R^{7}\right)$ in 5c, $\mathbf{d}$ und den entsprechenden Benzyliden-Verbindungen $(E)-5 \mathbf{f}, \mathbf{g}$ zeigt an, daß auch $5 \mathbf{c}$ und $\mathbf{d}$ (E)-Konfiguration besitzen.

Tab. 3. ${ }^{1} \mathrm{H}-\mathrm{NMR}$-Spektren der cyclischen Additionsprodukte 5 in $\mathrm{CCl}_{4}[\delta$, Multiplizität, $J(\mathrm{~Hz})]$

\begin{tabular}{|c|c|c|c|c|c|c|c|c|}
\hline & $\mathrm{R}^{1}$ & $\mathrm{R}^{2}$ & $\mathrm{R}^{3}$ & $6-\mathrm{H}$ & $\mathrm{R}^{4}$ & $R^{5}$ & $R^{6}$ & $\mathrm{R}^{7}$ \\
\hline$(E)-5 c$ & $\begin{array}{l}2.28 \\
t, 1\end{array}$ & $\begin{array}{l}1.22 \\
\mathrm{~s}\end{array}$ & $=\mathrm{R}^{2}$ & $\begin{array}{l}1.93 \\
\mathrm{mc}\end{array}$ & $\begin{array}{l}5.7 \\
\mathrm{mc}\end{array}$ & $\begin{array}{l}5.7 \\
\mathrm{mc}\end{array}$ & $=\mathrm{R}^{7}$ & $\begin{array}{l}2.98 \\
\mathrm{mc}\end{array}$ \\
\hline$(E)-\mathbf{5 d}$ & $\begin{array}{l}2.29 \\
t, 1\end{array}$ & $\begin{array}{l}1.21 \\
\mathrm{~s}\end{array}$ & $=\mathrm{R}^{2}$ & $\begin{array}{l}1.88 \\
\mathrm{mc}\end{array}$ & $\begin{array}{l}1.69 \\
\mathrm{mc}\end{array}$ & $\begin{array}{l}5.40 \\
\mathrm{mc}\end{array}$ & $=\mathrm{R}^{7}$ & $\begin{array}{l}2.95 \\
\mathrm{mc}\end{array}$ \\
\hline$(E)-\mathbf{5} \mathbf{f}$ & $\begin{array}{l}7.16 \\
\mathrm{mc}\end{array}$ & $\begin{array}{l}0.83 \\
\mathrm{~s}\end{array}$ & $=\mathrm{R}^{2}$ & $\begin{array}{l}1.88 \\
\mathrm{mc}\end{array}$ & $\begin{array}{l}5.62 \\
\mathrm{mc}\end{array}$ & $\begin{array}{l}5.62 \\
\mathrm{mc}\end{array}$ & $=\mathrm{R}^{7}$ & $\begin{array}{l}3.19 \\
\mathrm{mc}\end{array}$ \\
\hline$(E)-5 \mathrm{~g}$ & $\begin{array}{l}7.18 \\
\mathrm{mc}\end{array}$ & $\begin{array}{l}0.83 \\
s\end{array}$ & $=\mathrm{R}^{2}$ & $\begin{array}{l}1.82 \\
\mathrm{mc}\end{array}$ & $\begin{array}{l}1.67 \\
\mathrm{mc}\end{array}$ & $\begin{array}{l}5.38 \\
\mathrm{mc}\end{array}$ & $=\mathrm{R}^{7}$ & $\begin{array}{l}3.17 \\
\mathrm{mc}\end{array}$ \\
\hline$(E)-5 \mathrm{~h}$ & $\begin{array}{l}7.15 \\
s\end{array}$ & $\begin{array}{l}1.07 \\
\mathrm{~s}\end{array}$ & $\begin{array}{l}0.61 \\
s\end{array}$ & $\begin{array}{l}1.78-2.0 \\
\mathrm{~m}\end{array}$ & $\begin{array}{l}5.61 \\
\mathrm{mc}\end{array}$ & $\begin{array}{l}5.61 \\
\mathrm{mc}\end{array}$ & $\begin{array}{l}1.33 \\
\mathrm{~d}, 7\end{array}$ & $\begin{array}{l}3.60 \\
\text { br. q, } 7\end{array}$ \\
\hline$(E)-5 \mathbf{i}$ & $\begin{array}{l}7.22 \\
s\end{array}$ & $\begin{array}{l}0.81 \\
\mathrm{~s}\end{array}$ & $=\mathrm{R}^{2}$ & $\begin{array}{l}1.83 \\
\mathrm{mc}\end{array}$ & $\begin{array}{l}1.66 \\
\mathrm{mc}\end{array}$ & $\begin{array}{l}1.66 \\
\mathrm{mc}\end{array}$ & $=\mathrm{R}^{7}$ & $\begin{array}{l}3.10 \\
\mathrm{mc}\end{array}$ \\
\hline$(E)-5 \mathbf{j}$ & $\begin{array}{l}7.15 \\
s\end{array}$ & $\begin{array}{l}0.81 \\
S\end{array}$ & $=\mathrm{R}^{2}$ & verdeckt & $\begin{array}{l}1.4- \\
\mathrm{m}\end{array}$ & 2.2 & $=\mathrm{R}^{7}$ & $\begin{array}{l}3.01 \\
\mathrm{mc}\end{array}$ \\
\hline$(E)-5 \mathrm{n}$ & $\begin{array}{l}7.21 \\
\mathrm{~s}\end{array}$ & $\begin{array}{l}7.03 \\
\mathrm{mc}\end{array}$ & $\begin{array}{l}3.87 \\
\mathrm{mc}\end{array}$ & $\begin{array}{l}2.2-2.55 \\
\mathrm{~m}\end{array}$ & $\begin{array}{l}1.71 \\
\mathrm{mc}\end{array}$ & $\begin{array}{l}5.32 \\
\mathrm{mc}\end{array}$ & $\begin{array}{l}2.63 \\
\text { AB-System }\end{array}$ & $\begin{array}{l}3.32 \\
1, J=20\end{array}$ \\
\hline$(E)-50-t$ & $\begin{array}{l}6.55- \\
\mathrm{m}\end{array}$ & 7.1 & $\begin{array}{l}3.75^{b)} \\
m c\end{array}$ & $\begin{array}{l}1.85-2.85 \\
\mathrm{~m}\end{array}$ & $\begin{array}{l}5.35- \\
\mathrm{m}\end{array}$ & 6.2 & $\begin{array}{l}1.42 \\
d, 7\end{array}$ & $\begin{array}{l}3.60 \\
\text { br. q, 6b) }\end{array}$ \\
\hline$(E)-50-c^{\text {a) }}$ & $\begin{array}{l}7.22 \\
\mathrm{~s}\end{array}$ & $\begin{array}{l}7.01 \\
\mathrm{~s}\end{array}$ & $\begin{array}{l}4.06 \\
\mathrm{mc}\end{array}$ & $\begin{array}{l}2.1-3.1 \\
\mathrm{~m}\end{array}$ & $\begin{array}{l}5.3- \\
\mathrm{m}\end{array}$ & 6.0 & $\begin{array}{l}0.67 \\
d, 7\end{array}$ & $\begin{array}{l}3.45 \\
\mathrm{mc}\end{array}$ \\
\hline$(Z)-50-t$ & $\begin{array}{l}7.24 \\
s\end{array}$ & $\begin{array}{l}7.15 \\
s\end{array}$ & $\begin{array}{l}4.44 \\
\mathrm{dd}, 5,3\end{array}$ & $\begin{array}{l}2.5 \\
\mathrm{mc}\end{array}$ & $\begin{array}{l}5.4- \\
\mathrm{m}\end{array}$ & 6.0 & $\begin{array}{l}0.87 \\
d, 7\end{array}$ & $\begin{array}{l}3.25 \\
\mathrm{mc}\end{array}$ \\
\hline$(Z)-50-c^{a)}$ & $\begin{array}{l}7.3 \\
\mathrm{mc}\end{array}$ & & $\begin{array}{l}4.82 \\
\mathrm{mc}\end{array}$ & & & & $\begin{array}{l}0.36 \\
d, 7\end{array}$ & $\begin{array}{l}\approx 2.8 \\
\mathrm{mc}\end{array}$ \\
\hline$\left.(E)-5 \mathrm{p}-t^{\mathrm{a}}\right)$ & $\begin{array}{l}6.6- \\
m\end{array}$ & 7.5 & $\begin{array}{l}3.82 \\
\text { b) }\end{array}$ & $\begin{array}{l}1.7-2.9 \\
\mathrm{~m}\end{array}$ & $\begin{array}{l}1.52 \\
\text { br. s }\end{array}$ & $\begin{array}{l}5.7 \\
\mathrm{mc}\end{array}$ & $\begin{array}{l}1.39 \\
d, 7\end{array}$ & $\begin{array}{l}3.63 \\
b)\end{array}$ \\
\hline$\left.(E)-5 \mathrm{p}-c^{a}\right)$ & $\begin{array}{l}7.38 \\
s\end{array}$ & $\begin{array}{l}7.07 \\
S\end{array}$ & $\begin{array}{l}4.10 \\
\text { br.t, } 4\end{array}$ & $\begin{array}{l}2.47 \\
\mathrm{mc}\end{array}$ & $\begin{array}{l}1.87 \\
\text { br. s }\end{array}$ & $\begin{array}{l}5.2-5.5 \\
\mathrm{~m}\end{array}$ & $\begin{array}{l}0.64 \\
d, 7\end{array}$ & $\begin{array}{l}3.5 \\
\mathrm{mc}\end{array}$ \\
\hline$(Z)-5 \mathrm{p}-1$ & $\begin{array}{l}7.30 \\
s\end{array}$ & $\begin{array}{l}7.22 \\
\mathrm{~s}\end{array}$ & $\begin{array}{l}4.44 \\
d d, 5,2\end{array}$ & $2.7,2.1^{c)}$ & $\begin{array}{l}1.54 \\
\text { br. s }\end{array}$ & $\begin{array}{l}5.5 \\
\text { br. d, } 6\end{array}$ & $\begin{array}{l}0.84 \\
d, 7\end{array}$ & $\begin{array}{l}3.27 \\
\mathrm{mc}\end{array}$ \\
\hline$(Z)-5 p-c^{a)}$ & & & $\begin{array}{l}4.87 \\
\mathrm{mc}\end{array}$ & & & $\begin{array}{l}5.0-5.2 \\
\mathrm{~m}\end{array}$ & $\begin{array}{l}0.33 \\
d, 7\end{array}$ & \\
\hline
\end{tabular}

a) Identifizierung im Gemisch. - b) Teilweise verdeckt. - c) AB-Teil eines ABX-Systems, $J_{\mathrm{AB}} \approx 15 \mathrm{~Hz}$.

Die vier Diastereomeren von 50 und $\mathbf{p}$ lassen sich ebenfalls durch ihre ${ }^{1} \mathrm{H}-\mathrm{NMR}$-Spektren unterscheiden. Bei zwei 5 o-Isomeren zeigt 3-H eine ähnliche chemische Verschiebung wie bei $(E)-5 \mathbf{h}$ $(\delta=3.60,3.45$ und 3.60$)$, so daß diesen Verbindungen $(E)$-Konfiguration zukommt. In Übereinstimmung mit den Befunden an entsprechenden Bicyclen ${ }^{1,2)}$ sind diese Protonen in den betreffenden $(Z)$-Verbindungen um 0.35 bzw. 0.65 ppm stärker abgeschirmt. Im Einklang damit absorbiert $5-\mathrm{H}$ in den $(E)$-Isomeren bei höherem Feld als in den $(Z)$-Isomeren, weil es im ersten Fall zum Phenyl, im zweiten Fall zum Chlor cis steht. 


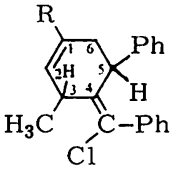

$\mathrm{R}=\mathrm{H} \quad(E)-50-t$

$\mathrm{R}=\mathrm{CH}_{3}(E)-5 \mathrm{p}-t$

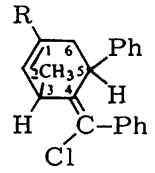

(E)-5o-c

(E) $-\mathbf{5 p}-c$<smiles>[R]c1cc(C)c(C(Cl)C(Cl)c2ccccc2)c(C(Cl)I)c1</smiles>

(Z) $-50-t$

$(Z)-5 p-t$

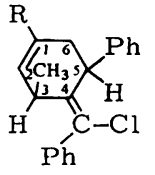

(Z) $-50-c$

(Z) $-\mathbf{5 p}-c$

Die ungewöhnliche Hochfeldverschiebung des Signals der Methylgruppe $\mathrm{R}^{6}$ in einem der $(E)$ Isomeren $(\delta=0.67)$ läßt sich mit der Annahme erklären, daß diese Methylgruppe in den positiven Abschirmbereich der 5-Phenylgruppe gelangt, was in $(E)-50-c$ möglich ist. Auf den gleichen Effekt läßt sich die unterschiedliche chemische Verschiebung der Methylgruppen in den beiden (Z)-Isomeren zurückführen: die Verbindung mit der stärker abgeschirmten Methylgruppe $(\delta=$ $0.36) \mathrm{mu}$ cis-konfiguriert sein $((Z)-50-c)$.

Wie Tab. 3 zeigt, besitzen die vier Diastereomeren 5p ${ }^{1} \mathrm{H}-\mathrm{NMR}$-Spektren, die sich von den korrespondierenden 5o-Isomeren lediglich durch die zusätzliche Methylgruppe unterscheiden.

Das 2:1-Produkt 6, das nach Massenspektrum aus zwei Einheiten $2 \mathrm{f}$ und einem Molekül 1c aufgebaut ist, zeigt im ${ }^{13} \mathrm{C}$-NMR-Spektrum außer den Aromatenpeaks noch vier Vinylkohlenstof$\mathrm{fe}$, so daß es sich aufgrund der Zahl der Doppelbindungsäquivalente um einen Bicyclus handeln muß. Verbindung 6, deren Konstitution aus mechanistischen Überlegungen erhalten wurde, liegt bevorzugt in der angegebenen Konformation vor, wie ihr $200-\mathrm{MHz}-{ }^{1} \mathrm{H}-\mathrm{NMR}$-Spektrum zeigt ${ }^{4}$.

Tab. 4. 'H-NMR-chemische Verschiebungen $\delta$ (Diagonale) und Kopplungskonstanten $(\mathrm{Hz})$ des 2:1-Produkts 6 in $\mathrm{CDCl}_{3}$

\begin{tabular}{ccccccccc}
\hline & & & $\downarrow$ & & $\downarrow$ & $\downarrow$ & $\downarrow$ & $\downarrow$ \\
& $3-\mathrm{H}_{\mathrm{a}}$ & $3-\mathrm{H}_{\mathrm{e}}$ & $4-\mathrm{H}_{\mathrm{a}}$ & $5-\mathrm{H}_{\mathrm{a}}$ & $5-\mathrm{H}_{\mathrm{e}}$ & $6-\mathrm{H}_{\mathrm{a}}$ & $7-\mathrm{H}_{\mathrm{a}}$ & $10-\mathrm{H}$ \\
\hline $3-\mathrm{H}_{\mathrm{a}}$ & 1.12 & 13.1 & 11.9 & - & - & - & - & - \\
$3-\mathrm{H}_{\mathrm{c}}$ & 13.1 & 1.21 & 3.7 & - & 1.9 & - & - & - \\
$4-\mathrm{H}_{\mathrm{a}}$ & 11.9 & 3.7 & 2.53 & 11.9 & 3.7 & - & - & 8.7 \\
$5-\mathrm{H}_{\mathrm{a}}$ & - & - & 11.9 & 0.83 & 12.5 & 12.3 & - & - \\
$5-\mathrm{H}_{\mathrm{c}}$ & - & 1.9 & 3.7 & 12.5 & 2.21 & 5.2 & - & - \\
$6-\mathrm{H}_{\mathrm{a}}$ & - & - & - & 12.3 & 5.2 & 2.83 & 9.3 & - \\
$7-\mathrm{H}_{\mathrm{a}}$ & - & - & - & - & - & 9.3 & 3.69 & - \\
$10-\mathrm{H}$ & - & - & 8.7 & - & - & - & - & 4.92 \\
\hline
\end{tabular}

Methylgruppen bei $\delta=0.50,0.87,0.97,1.08,1.66,1.69$.

Tab. 4 enthält die vollständige Analyse des 'H-NMR-Spektrums von 6. Mit Ausnahme von 3- $\mathrm{H}_{\mathrm{a}}$ und $-\mathrm{H}_{\mathrm{e}}$, die mit 4-H und $5-\mathrm{H}_{\mathrm{e}}$ ein ABXY-System bilden, gilt für alle anderen Spinsysteme $\Delta v>10 \mathrm{~J}$, so daß das ganze Spektrum nach 1 . Ordnung ausgewertet werden kann. Wie für Cyclohexane zu erwarten, sind alle Kopplungen $J_{\mathrm{aa}}$ in der Größenordnung von $12 \mathrm{~Hz}$, während die Axial-Äquatorial-Kopplungen $3-5 \mathrm{~Hz}$ betragen. Zusätzlich findet man noch eine Fernkopplung von $1.9 \mathrm{~Hz}$ zwischen den W-förmig angeordneten Protonen $3-\mathrm{H}_{\mathrm{e}}$ und $5-\mathrm{H}_{\mathrm{e}}$. Die in Tab. 4 durch Pfeile angegebenen Doppelresonanzexperimente bestätigen die dort getroffene Zuordnung. Strahlt man beispielsweise die Resonanz bei $\delta=2.83$ ein, erkennt man aus Kolonne 6- $\mathrm{H}_{\mathrm{a}}$, daß dadurch die Resonanzen von 3- $\mathrm{H}_{\mathrm{a}}, 3-\mathrm{H}_{\mathrm{e}}, 4-\mathrm{H}$ und 10-H nicht verändert werden. Das Quartett von $5-\mathrm{H}_{\mathrm{a}}$ wird zu einem Triplett, das zunächst komplizierte Multiplett von $5-\mathrm{H}_{\mathrm{e}}$ zu einem Dublett von Dubletts von Dubletts $(J=12.5,3.7,1.9 \mathrm{~Hz})$ und das Dublett von $7-\mathrm{H}$ zu einem Singulett. In gleicher Weise wurden alle anderen Kopplungsbeziehungen überprüft. 
Die Position des Chlors läßt sich aus der Größe der Kopplung von 7- $\mathrm{H}$ mit 6-H $\left(J_{\mathrm{aa}}=9.3 \mathrm{~Hz}\right)$ entnehmen. Die Vinylgruppierung an C-4 muß schließlich in äquatorialer Stellung stehen, weil 4- $\mathrm{H}$ eine Kopplung von $11.9 \mathrm{~Hz}$ zu 3- $\mathrm{H}_{\mathrm{a}}$ und $5-\mathrm{H}_{\mathrm{a}}$ hat, was nur durch eine Axial-Axial-Kopplung erklärbar ist.

\section{Diskussion}

a) Bildungsweise der 1: 1-Produkte 3-5

Die Bildung der Allylchloride 3 und 4 sowie der Cyclohexene 5 wird in Schema 2 am Beispiel der Addition des Trimethylpropargylchlorids $1 \mathbf{b}$ an 1,3-Butadien (2a) skizziert . Das zugehörige Energieprofil findet sich in Abb. 1.

Schema 2
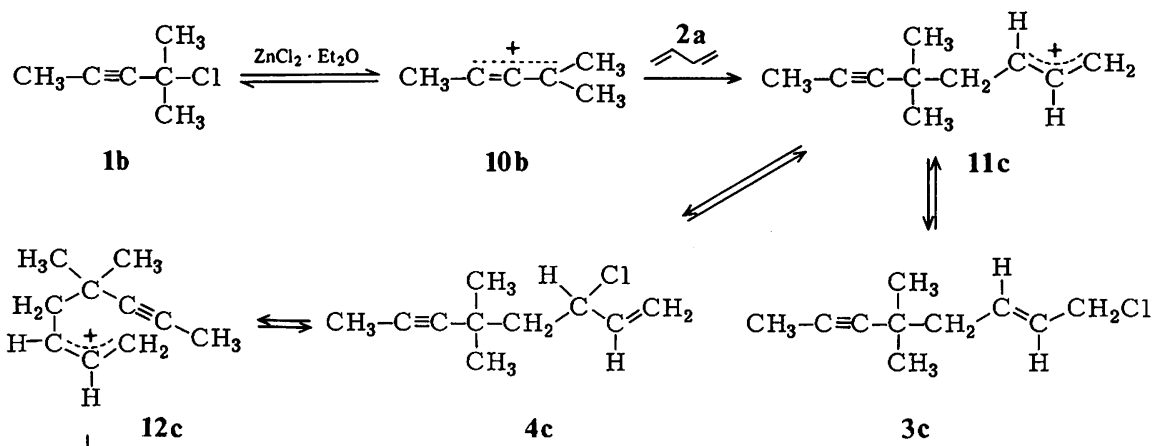<smiles>C[C+]=C1CC=CCC1(C)C</smiles>

$13 \mathrm{c}$

$(E)-5 c$<smiles>CC#CC(C)(C)C/C=C/CCl</smiles>

$14 \mathrm{c}$

Zunächst dissoziiert das Propargylchlorid 1b unter Bildung des Allenyl-Kations 10b, das sich an s-trans-Butadien addiert und das exo-substituierte Allyl-Kation 11c ergibt. Das Chlorid-Ion kann nun an beiden Enden des Allyl-Kations angreifen, wobei die als Primärprodukte isolierten Allylchloride 3c und 4c entstehen. Nach Abb. 1 hat die Umlagerung von $4 \mathbf{c}$ zu $3 \mathbf{c}$ über das Allyl-Kation 11 eine niedrigere Aktivierungsenergie als die Cyclisierung, so daß bei schonendem Behandeln des 3c/4c-Gemisches mit LewisSäure das 1,2-Additionsprodukt $\mathbf{4 c}$ in das thermodynamisch stabilere $\mathbf{3 c}$ übergeht.

Die Umlagerung von 3c zu 5c muß über das Allylchlorid 4c als Zwischenstufe verlaufen. Im Gegensatz zu 3c kann dieses Allylchlorid bei der Dissoziation außer 11c auch das endo-Allyl-Kation 12c ergeben, das die zur Cyclisierung geeignete Konfiguration besitzt. Bildung von 12c durch Rotation des Allyl-Kations 11c läßt sich ausschließen, weil hierbei ein energetisch ungünstiges orthogonales Allyl-Kation durchlaufen werden müßte. Das Vinyl-Kation 13c, das durch $\pi$-Cyclisierung von $12 \mathrm{c}$ entsteht, wird schließlich durch Chlorid von der sterisch weniger behinderten Seite aus angegriffen, so daß selektiv das $(E)$-Vinylchlorid 5c entsteht. 


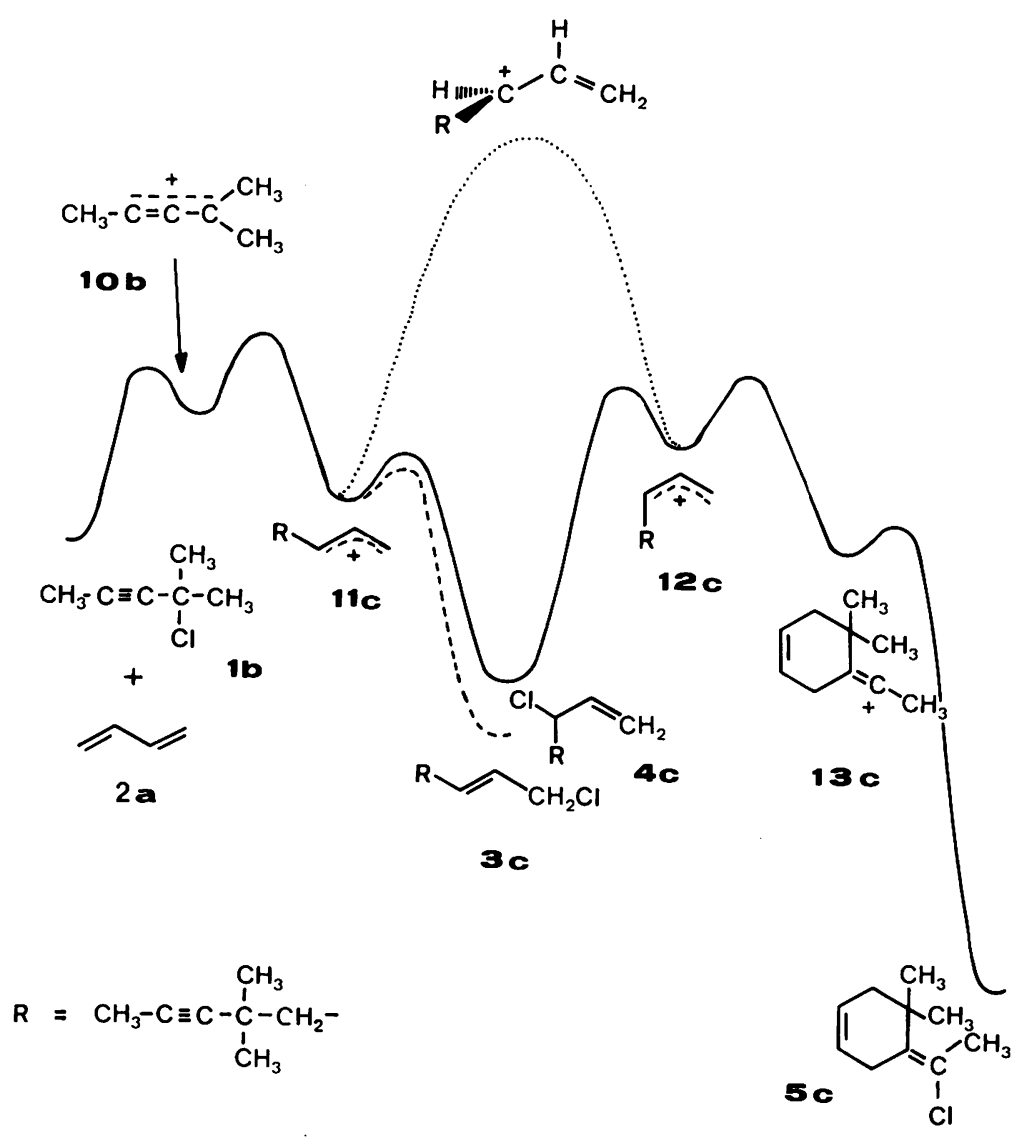

Abb. 1. Schematisches Energieprofil der Lewis-Säure-katalysierten Umsetzung des Propargylchlorids $\mathbf{1 b}$ mit 1,3-Butadien

\section{b) Struktur der linearen Additionsprodukte}

Die isolierten Allylchloride enthalten ausnahmslos eine Acetylen-Einheit und nicht eine Allen-Einheit. Damit ist die störungstheoretische Vorhersage, daß AllenylKationen von $\pi$-Systemen am $\mathrm{sp}^{2}$-Kohlenstoff, der Position mit dem größten LUMOKoeffizienten, angegriffen werden ${ }^{5)}$, wiederum bestätigt. Die Konstitution der Allylchloride 3 und 4 zeigt ferner, daß unsymmetrische Diene ausschließlich so angegriffen werden, daß intermediär die Allyl-Kationen mit der größten Zahl terminaler Alkylgruppen entstehen. Dieser Befund läßt sich sowohl mit der Größe der Koeffizienten im HOMO der Diene ${ }^{6}$ ) wie auch mit der unterschiedlichen Stabilisierung von AllylKationen durch 1- und 3- bzw. 2-ständige Alkylgruppen ${ }^{7)}$ in Einklang bringen.

Wie Tab. 1 zeigt, werden die 1,2-Additionsprodukte 4 neben den 1,4-Additionsprodukten 3 nur bei den Additionen an Butadien (2a) sowie bei der Umsetzung von $1 \mathbf{d}$ mit Piperylen (2c) gefunden. Wir nehmen an, daß zunächst in allen Systemen beide Addi- 
tionsprodukte 3 und 4 nebeneinander entstehen (kinetische Kontrolle). Im allgemeinen äquilibrieren diese Allylchloride in Gegenwart der Lewis-Säure unter bevorzugter Bildung der thermodynamisch stabileren Allyl-Isomeren 3. Diese Isomerisierung ist im Fall der Butadien-Addukte 4c und $\mathbf{4 f}$ langsamer, weil hierbei monoalkylierte AllylKationen als Zwischenstufen auftreten müssen, während die Umlagerung der anderen Allylsysteme über stabilere, höher alkylierte Allyl-Kationen 11 erfolgen kann. Die aus Piperylen (2c) entstehenden 1,2-Additionsprodukte enthalten wie die 1,4-Produkte eine trans-disubstituierte Doppelbindung, so daß hier das 1,4-Addukt im Gleichgewicht nicht eindeutig bevorzugt ist. Daher liefert die Umsetzung von 1d mit $2 \mathbf{c}$ ein Gemisch aus 30 und 4o. Vermutlich sind sterische Gründe dafür verantwortlich, daß $4 \mathbf{h}$ im Gleichgewicht neben $\mathbf{3 h}$ nur in geringer Konzentration vorliegt und NMR-spektroskopisch nicht nachweisbar ist.

\section{c) Struktur der cyclischen Produkte}

Das Allyl-Kation 12 hat prinzipiell vier verschiedene Möglichkeiten des elektrophilen Angriffs an der CC-Dreifachbindung, wie durch die Linien $\mathrm{a}-\mathrm{d}$ in Schema 3 angedeutet ist. Cyclisierung nach a und $\mathrm{b}$, die auch von den exo-Allyl-Kationen 11 ausgehen könnte, sollte nicht sehr günstig sein. Nach a muß die Cyclobutan-Ringspannung aufgebracht werden, und nach $b$ entsteht das 1-Cyclopentenyl-Kation 16, das wegen der starken Abweichung des Vinyl-Kation-Fragments von der linearen Idealgeometrie energetisch sehr hoch liegt ${ }^{8)}$.

Schema 3

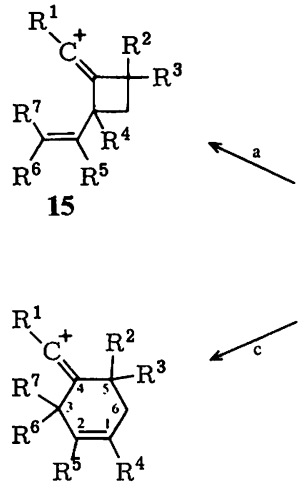

13

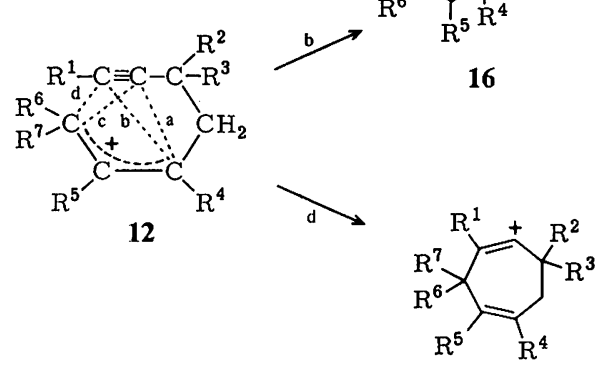

17

Ist $\mathrm{R}^{1}$ ein Phenylrest, sollte Cyclisierung nach $\mathrm{c}$ eindeutig bevorzugt sein, da im Vinyl-Kation 13 der Phenylrest direkt an das Carbenium-Ionen-Zentrum gelangt. Ist $\mathrm{R}^{1}$ ein Alkylrest, ist nur ein kleiner Energieunterschied zwischen den Kationen 13 und 17 zu erwarten. Der in $\mathbf{1 7}$ vorliegende Siebenring hat eine etwas größere Ringspannung als der sechsgliedrige Ring in 13. Außerdem sind Vinyl-Kationen im siebengliedrigen Ring im Vergleich zu entsprechend substituierten acyclischen Systemen etwas destabilisiert ${ }^{9}$, so daß auch für $\mathrm{R}^{1}=\mathrm{CH}_{3}$ ein geringer Vorzug von 13 gegenüber 17 zu erwarten ist. In 
Übereinstimmung damit liefern Propargylchloride $1\left(\mathrm{R}^{1}=\mathrm{CH}_{3}, \mathrm{Ph}\right)$ mit acyclischen Dienen als einzige cyclische Produkte die Vinylchloride 5, die sich von 13 ableiten.

Die Konfiguration an der exocyclischen Doppelbindung von 5 läßt sich durch die Stereochemie des Vinyl-Kations 13 erklären: In allen Fällen außer 130 und $\mathbf{p}$ ist $C-5$ höher substituiert als $C-3$, so daß sich das Chlorid-Ion von der $R^{6}, R^{7}$-Seite her annähert und die $(E)$-Vinylchloride liefert. In 130 und $p$ tragen dagegen $C-3$ und $C-5$ je einen Substituenten, so daß hier der nucleophile Angriff von beiden Seiten aus erfolgt.

\section{d) Kinetische Befunde}

Obwohl viele der in Tab. 1 und im experimentellen Teil angegebenen Reaktionszeiten länger sind als für den vollständigen Ablauf der Umsetzungen tatsächlich erforderlich, lassen sich doch folgende relative Reaktivitäten entnehmen:

$$
\begin{array}{ll}
\text { Propargylchloride: } & \mathbf{1} \mathbf{a} \ll \mathbf{1 b}<\mathbf{1} \mathbf{c}, \mathbf{d} \\
\text { Diene: } & \mathbf{2} \mathbf{a}<\mathbf{2 b}-\mathbf{h}
\end{array}
$$

Daraus folgt, daß der Übergangszustand der Additionsreaktionen von der Stabilität der Allenyl-Kationen 10 wie auch von der Stabilität der Allyl-Kationen 11 abhängig ist. Der Befund, daß verschiedene Diene unterschiedlich rasch reagieren, bedeutet weiterhin, daß die Dissoziation der Propargylhalogenide reversibel ist.

Auch die Cyclisierungen verlaufen umso rascher, je besser Edukt- und ProduktKation stabilisiert sind. Beispielsweise erfordert die Cyclisierung des Butadien-Addukts 3f (monoalkyliertes Allylsystem) 24 Stunden bei $25^{\circ} \mathrm{C}$, die Cyclisierung von $3 \mathrm{~g}$ (dialkyliertes Allylsystem) 5 Stunden bei $-30^{\circ} \mathrm{C}$, und $3 \mathbf{p}$ (trialkyliertes Allylsystem) kann überhaupt nicht isoliert werden, weil es bei $-78^{\circ} \mathrm{C}$ spontan cyclisiert. Die Abhängigkeit der Cyclisierungsgeschwindigkeit von der Stabilität der dabei entstehenden Carbenium-lonen geht daraus hervor, daß die bei $-78^{\circ} \mathrm{C}$ aus $1 \mathrm{c}$ erhaltenen Reaktionsprodukte stets eine gewisse Menge an cyclischem Material enthalten, während aus 1b bei $-78^{\circ} \mathrm{C}$ ausschließlich lineare Additionsprodukte erhalten werden. Im einen Fall (1c) führt die $\pi$-Cyclisierung zu einem gut stabilisierten Phenylvinyl-Kation $13\left(R^{1}=\right.$ $\mathrm{Ph})$, während im anderen Fall (1b) ein weniger stabiles Vinyl-Kation $13\left(\mathrm{R}^{1}=\mathrm{CH}_{3}\right)$ gebildet wird.

Das intermediäre Auftreten freier endo-Allyl-Kationen 12 ist zur Bildung von 13 nicht unbedingt erforderlich. Der erwähnte Befund, daß $\mathbf{4} \mathbf{f}$ beim Behandeln mit LewisSäure cyclisiert und nicht zum anderen Allyl-Isomeren $3 \mathrm{f}$ umlagert, läßt sich am einfachsten durch eine anchimere Beteiligung der Dreifachbindung bei der Ionisation von $4 \mathrm{f}$ erklären. Im Fall $\mathrm{R}^{1}=\mathrm{CH}_{3}$ ist die Beteiligung der Dreifachbindung weniger wichtig, denn die Umlagerung von $4 \mathbf{c}$ zu $3 \mathbf{c}$ über das Allyl-Kation 11c ist rascher als der möglicherweise anchimer unterstützte Prozeß $\mathbf{4 c} \rightarrow \mathbf{5 c}$.

Alle Umsetzungen von $1 \mathrm{c}$ mit 1,3-Dienen lieferten bereits bei $-78^{\circ} \mathrm{C}$ teilweise cyclisches Material 5, dessen Anteil von der Reaktionsdauer unabhängig ist. Da wir weiterhin zeigen konnten, daß $3 \mathrm{~g}$ bei $-60^{\circ} \mathrm{C}$ unter den Bedingungen der Additionsreaktion noch nicht zu $5 \mathrm{~g}$ umlagert, können die bei $-78^{\circ} \mathrm{C}$ gebildeten Vinylchloride $5 \mathrm{f}-\mathbf{i}$ nicht aus den Allylchloriden $\mathbf{3 f}-\mathbf{i}$ hervorgehen. Möglicherweise entstehen sie durch Angriff von $10 \mathrm{c}$ an $s$-cis-Dienen, wobei über das endo-Allyl-Kation 12 direkt das Vinyl-Kation 
13 gebildet wird ${ }^{10)}$. Damit in Einklang steht die ausschließliche Bildung von $5 \mathbf{j}$ aus 1c und dem $s$-cis-fixierten Dien 2 e bei $-40^{\circ} \mathrm{C}$.

\section{e) Wann ist die Bildung von 1: 1-Additionsprodukten möglich?}

Mechanistische Überlegungen führten uns kürzlich zu der Schlußfolgerung, daß Lewis-Säure-katalysierte Additionen von Alkylhalogeniden an CC-Mehrfachbindungen nur dann zu 1:1-Produkten führen können, wenn die Edukte rascher dissoziieren als die Produkte ${ }^{11)}$. Mit Hilfe der Solvolysekonstanten der Propargylchloride 1a-d und einiger Allylchloride, die als Modelle für die Additionsprodukte $\mathbf{3}$ und $\mathbf{4}$ dienen (Tab. 5), lassen sich nun die Ergebnisse der Tab. 1 interpretieren.

Tab. 5. Solvolysekonstanten ungesättigter Alkylhalogenide in 80 proz. Ethanol/Wasser bei $25^{\circ} \mathrm{C}$

\begin{tabular}{clcc}
\hline & & $k_{1}, \mathrm{~s}^{-1}$ & Lit. \\
\hline $\mathbf{1 a}$ & $\mathrm{H}-\mathrm{C} \equiv \mathrm{C}-\mathrm{C}\left(\mathrm{CH}_{3}\right)_{2} \mathrm{Cl}$ & $2.4 \times 10^{-7}$ & $12)$ \\
$\mathbf{1 8}$ & $\mathrm{H}_{2} \mathrm{C}=\mathrm{CH}-\mathrm{CH}\left(\mathrm{CH}_{3}\right) \mathrm{Cl}$ & $4.9 \times 10^{-7}$ & $13)$ \\
$\mathbf{1 9}$ & $\mathrm{CH}_{3}-\mathrm{CH}=\mathrm{CH}-\mathrm{CH}_{2} \mathrm{Cl}$ & $\approx 1 \times 10^{-6}$ & a) \\
$\mathbf{2 0}$ & $\left(\mathrm{CH}_{3}\right)_{2} \mathrm{C}=\mathrm{CH}-\mathrm{CH}_{2} \mathrm{Cl}$ & $\approx 4 \times 10^{-4}$ & b) \\
$\mathbf{1 b}$ & $\mathrm{CH}_{3}-\mathrm{C} \equiv \mathrm{C}-\mathrm{C}\left(\mathrm{CH}_{3}\right)_{2} \mathrm{Cl}$ & $5.1 \times 10^{-4}$ & $12)$ \\
$\mathbf{1 c}$ & $\mathrm{C}_{6} \mathrm{H}_{5}-\mathrm{C} \equiv \mathrm{C}-\mathrm{C}\left(\mathrm{CH}_{3}\right)_{2} \mathrm{Cl}$ & $2 \times 10^{-3}$ & c) \\
$\mathbf{2 1}$ & $\mathrm{CH}_{3} \mathrm{CH}=\mathrm{CH}-\mathrm{CH}\left(\mathrm{CH}_{3}\right) \mathrm{Cl}$ & $\approx 3.8 \times 10^{-3}$ & d) \\
$\mathbf{1 d}$ & $\mathrm{C}_{6} \mathrm{H}_{5}-\mathrm{C} \equiv \mathrm{C}-\mathrm{CH}\left(\mathrm{C}_{6} \mathrm{H}_{5}\right) \mathrm{Cl}$ & $5.9 \times 10^{-2}$ & c) \\
22 & $\left(\mathrm{CH}_{3}\right)_{2} \mathrm{C}=\mathrm{CH}-\mathrm{CH}\left(\mathrm{CH}_{3}\right) \mathrm{Cl}$ & $\approx 4$ & e) \\
\hline
\end{tabular}

a,b) Schätzung aus $k_{1}(18)$ und dem Geschwindigkeitsverhältnis von $2.1^{\text {a) }}$ und $760^{\text {b) }}$, das für 80proz. Ethanol errechnet wurde ${ }^{14)}$. - c) Konduktometrische Messungen in $10^{-3} \mathrm{M}$ Lösung; $T$. W. Bentley, unveröffentlicht. - d) Aus Daten in Lit. ${ }^{15)}$ unter Verwendung der $m y$-Gleichung berechnet. - e) Schätzung aus $k_{1}(21)$ und dem Geschwindigkeitsverhältnis der entsprechenden Allylbromide in 80proz. Ethanol (1133) ${ }^{16)}$.

1 a dissoziiert langsamer als mono- und dialkylsubstituierte Allylchloride (Tab. 5) und kann daher weder an Butadien (2a) noch an Isopren (2b) addiert werden. Einmal gebildetes $\mathbf{3 a}, \mathbf{b}$ oder $\mathbf{4 a}, \mathbf{b}$ addiert sich nämlich rascher an weiteres Dien als $\mathbf{1 a}$, so daß Polymerisation von 2a bzw. 2 b erfolgt. Der Unterschied der Dissoziationsgeschwindigkeiten von Edukten und Produkten ist hier in Wirklichkeit noch größer als aus Tab. 5 hervorgeht, da 1 a unter Lewis-Säure-Katalyse in 1-Chlor-3-methyl-1,2-butadien umlagert, das etwa $45 \mathrm{mal}$ langsamer dissoziiert als $1 \mathbf{a}^{17)}$.

Das Trimethylpropargylchlorid $1 \mathbf{b}$ bildet mit Butadien (2a) und Isopren (2b) 1:1Additionsprodukte, da 1b rascher dissoziiert als 19 und 20, die Modellverbindungen für 3c und 3d. Vermutlich ist die geringe Ausbeute an 3d auf partielle Polymerisation des Isoprens zurückzuführen (Solvolysekonstanten sehr ähnlich). 22, die Modellverbindung für $\mathbf{3 e}$, dissoziiert viel rascher als $\mathbf{1 b}$, so daß bei der Umsetzung von $\mathbf{1 b}$ mit $\mathbf{2} \mathbf{f}$ nur höhermolekulare Produkte entstehen.

Das Propargylchlorid 1c liefert erwartungsgemäß mit Butadien (2a), Isopren (2b), 2,3-Dimethylbutadien (2d) und 1,2-Dimethylencyclohexan (2e) 1:1-Produkte, da 1c reaktiver ist als das Vinylchlorid 5j und die Allylchloride 19 und 20, die als Modelle für 3f und $\mathbf{3 g}$, i dienen. Das Entstehen von $3 \mathrm{~h}$ aus $1 \mathrm{c}$ und Piperylen (2c) ist aufgrund der 
Daten in Tab. 5 nicht vorhersagbar, da 21 (Modell für $\mathbf{3 h}$ ) etwas rascher solvolysiert als 1c. Möglicherweise ist der in Tab. 5 angegebene Wert für 21 falsch; andererseits kann aber auch von diesem qualitativen Modell ${ }^{11)}$ bei so geringen Unterschieden der Solvolysekonstanten keine zuverlässige Voraussage erwartet werden. Mit 2f-h bildet 1c Trialkyl- bzw. Tetraalkylallylsysteme 3, die wesentlich reaktiver sind (Modell 22) als 1c, so daß sie nicht isoliert werden können.

Die Bildung von 3n und 3o, 40 aus $1 \mathrm{~d}$ und den entsprechenden Dienen ist aufgrund der Daten in Tab. 5 zu erwarten. In Übereinstimmung mit der Voraussage auf der Grundlage der Solvolysekonstanten wird bei der Umsetzung von 1d mit $\mathbf{2 g}$ das Allylchlorid 3p nicht gefunden. Intramolekulare Cyclisierung von 3p führt jedoch rasch zum Vinylchlorid 5p, das als langsam dissoziierendes Produkt wieder „erlaubt“ ist. Da das Verhältnis von Cyclisierung zu Polymerisation (d.h. Reaktion von 3p mit 2g) umgekehrt proportional zur Konzentration des Diens ist, wurde die angegebene Ausbeute an $\mathbf{5 p}$ durch langsames Zutropfen von $\mathbf{2 g}$ und $1 \mathrm{~d}$ zum Katalysator erreicht. Es ist anzunehmen, daß bei gleicher Zugabeweise von $2 \mathrm{~g}$ und $1 \mathrm{c}$ zum Katalysator die Stationärkonzentration an Dien $\mathbf{2 g}$ wegen der geringeren Reaktivität von $1 \mathrm{c}$ höher ist als im vorigen Versuch, so daß hier die intermolekularen Folgereaktionen (Polymerisation) rascher sind als die intramolekulare Cyclisierung.

\section{f) Bildungsweise des 2: 1-Produkts 6}

Schema 4 zeigt, daß bei der Umsetzung von 1 c mit 2 f zunächst das lineare Additionsprodukt 3k entsteht. Dieses dissoziiert jedoch rascher als 1c (22/1c, Tab. 5) und reagiert daher schneller mit 2 f als 1c. Dabei entsteht das Allyl-Kation 25, das durch stufenweise intramolekulare [3 3 2]-Cycloaddition in das sekundäre Carbenium-Ion 24 übergeht ${ }^{18)}$. Anlagerung von Chlorid liefert schließlich das 2:1-Addukt 6, das langsamer dissoziiert als 1c und deshalb isoliert werden kann. Die geringe Ausbeute an 6 (23\%) erklären wir dadurch, daß 25 auch mit weiterem Dien unter Bildung höhermolekularer Produkte reagieren kann oder daß eine 1,2-Hydridverschiebung in 24 zu einem Allyl-Kation führt, das ebenfalls mit weiterem $\mathbf{2} \mathbf{f}$ reagieren sollte.

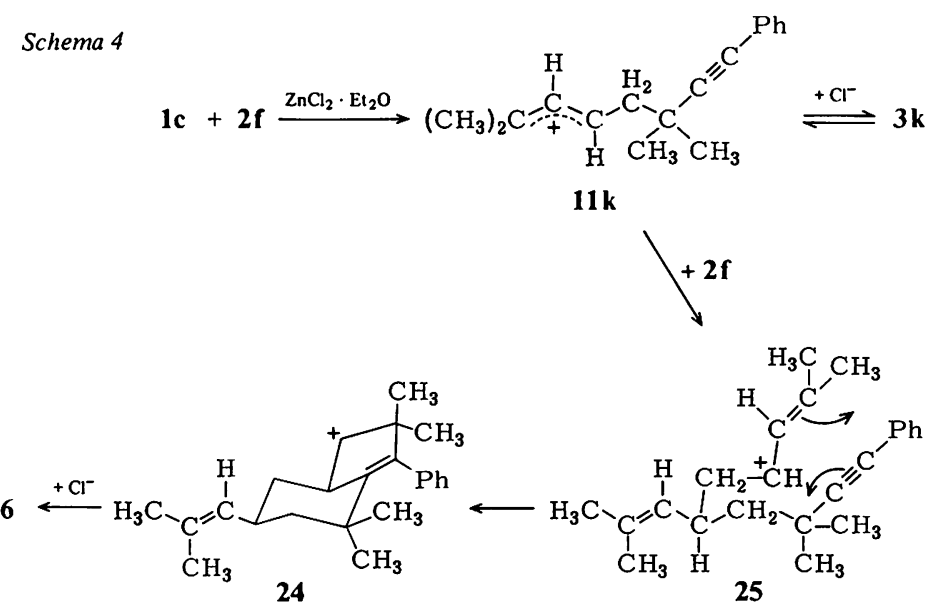




\section{Schlußfolgerungen}

Die stufenweise Cycloaddition, die bei den Reaktionen von Allenyl-Kationen mit cyclischen Dienen postuliert worden ist ${ }^{1,2)}$, kann bei den hier untersuchten Umsetzungen mit acyclischen 1,3-Dienen durch die Isolierung linearer Zwischenprodukte eindeutig nachgewiesen werden. Aus dem Vergleich der Reaktionsgeschwindigkeiten ergibt sich, daß der Übergangszustand der intermolekularen Additionen wie auch der intramolekularen Cyclisierungen die Stabilität der Edukt- wie auch der ProduktCarbenium-Ionen reflektiert. Infolgedessen läßt sich auch die Regioselektivität des elektrophilen Angriffs an unsymmetrischen Dienen und die Regioselektivität der Cyclisierung durch die relative Stabilität der potentiellen Produkt-Carbenium-Ionen erklären.

Die relativen Solvolysekonstanten von Edukten und Produkten in 80proz. Ethanol ermöglichten wiederum eine recht zuverlässige Voraussage, in welchen Fällen 1:1Additionsprodukte gebildet werden können. Schwierig wird eine derartige Analyse ${ }^{11)}$ jedoch in solchen Fällen, in denen das primäre Additionsprodukt rasche intramolekulare Folgereaktionen eingeht, weil dann nicht mehr von vorneherein klar ist, welche Solvolysekonstanten für die Produkte einzusetzen sind.

Wir danken Dr. T. W. Bentley, University College of Swansea, für die Bestimmung der Solvolysekonstanten von 1c und d. Der Deutschen Forschungsgemeinschaft und dem Fonds der Chemischen Industrie danken wir für finanzielle Unterstützung dieser Arbeit.

\section{Experimenteller Teil}

IR-Spektren: Beckman Acculab 1 und 3; innerhalb einer Gruppe sind die IR-Banden nach fallender Intensität geordnet. - ${ }^{1} \mathrm{H}-\mathrm{NMR}$-Spektren: JEOL JNM-C-60-HL und JEOL PMX-60. ${ }^{13}$ C-NMR-Spektren: JEOL JNM-PS-100. - UV-Spektren: Leitz-Unicam SP 800. - Massenspektren: Varian MAT CH 4 und Varian MAT 311A. - Die Schmelzpunkte sind nicht korrigiert.

1. 3-Chlor-3-methyl-1-butin (1 a) und 1,3-Butadien (2a): Eine Lösung von $2.04 \mathrm{~g} \mathrm{(19.9} \mathrm{mmol)}$ $1 \mathrm{a}$ in $10 \mathrm{ml} \mathrm{CH} \mathrm{Cl}_{2}$ wurde unter Rühren zu einer auf $-78^{\circ} \mathrm{C}$ gekühlten Lösung von $4.50 \mathrm{~g}(83.2$ mmol) $2 \mathrm{a}$ und $3.9 \mathrm{~g} \mathrm{ZnCl}_{2}$ in $4.6 \mathrm{ml}$ Ether und $9.3 \mathrm{ml} \mathrm{CH}_{2} \mathrm{Cl}_{2}$ getropft. Nach $7 \mathrm{~d}$ bei $-78^{\circ} \mathrm{C}$ wurde $\frac{1}{3}$ der Probe auf wäßr. Ammoniak gegossen, das $\mathrm{ZnCl}_{2}$ ausgewaschen, die organische Phase getrocknet und 2a sowie ein Teil des $\mathrm{CH}_{2} \mathrm{Cl}_{2}$ und Ethers i. Vak. abgezogen. Das ${ }^{1} \mathrm{H}$-NMR-Spektrum des Rückstandes zeigt außer $\mathrm{CH}_{2} \mathrm{Cl}_{2}$ und Ether ausschließlich $1 \mathrm{a}$.

Die verbleibenden $\frac{2}{3}$ der Probe wurden $2 \mathrm{~h}$ auf $0^{\circ} \mathrm{C}$ aufgewärmt, dann wie oben aufgearbeitet. Destillation lieferte $0.44 \mathrm{~g}$ 1-Chlor-3-methyl-1,2-butadien, $0.15 \mathrm{~g}$ nicht identifiziertes Material im Siedebereich $25-100^{\circ} \mathrm{C} / 1.5$ Torr und $1.07 \mathrm{~g}$ Rückstand.

2. 1a und Isopren (2 b): $2.04 \mathrm{~g}(19.9 \mathrm{mmol}) \mathbf{1 a}$ und $1.36 \mathrm{~g}(20.0 \mathrm{mmol}) 2 \mathrm{~b}$ wurden in $10 \mathrm{ml}$ $\mathrm{CH}_{2} \mathrm{Cl}_{2}$ zu einer Lösung von $1.0 \mathrm{~g} \mathrm{ZnCl}_{2}$ in $1.2 \mathrm{ml}$ Ether und $23 \mathrm{ml} \mathrm{CH}_{2} \mathrm{Cl}_{2}$ bei $-78^{\circ} \mathrm{C}$ getropft und $72 \mathrm{~h}$ bei dieser Temperatur belassen. $\frac{2}{3}$ der Probe wurden wie oben aufgearbeitet. Das gesamte Material war bei Raumtemp. bei 50 Torr flüchtig, so daß keine Reaktion erfolgt sein konnte.

$\frac{1}{3}$ der Probe wurde nach $2 \mathrm{~h}$ Erwärmen auf $0^{\circ} \mathrm{C}$ aufgearbeitet. Destillation lieferte $0.47 \mathrm{~g}$ 1-Chlor-3-methyl-1,2-butadien und $0.03 \mathrm{~g} \mathrm{1a}$, kein Material im Bereich $25-90^{\circ} \mathrm{C} / 0.1$ Torr und $0.55 \mathrm{~g}$ schwerflüchtigen Rückstand.

3. 4-Chlor-4-methyl-2-pentin (1 b) und 1,3-Butadien (2a): $\mathrm{Zu}$ einer Lösung aus $4.1 \mathrm{~g} \mathrm{ZnCl}_{2}$ in $5 \mathrm{ml}$ Ether und $10 \mathrm{ml} \mathrm{CH}_{2} \mathrm{Cl}_{2}$ wurden bei $-78^{\circ} \mathrm{C}$ nacheinander $4.50 \mathrm{~g}(83.2 \mathrm{mmol}) 2 \mathrm{a}$ und $2.32 \mathrm{~g}$

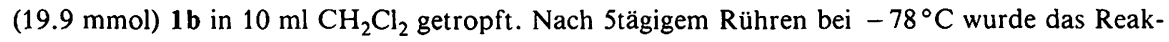

Chem. Ber. 115 (1982) 
tionsgemisch auf $100 \mathrm{ml}$ eisgekühltes konz. wäßr. Ammoniak gegeben und gründlich durchgeschüttelt. Die organische Phase wurde abgetrennt, nochmals mit konz. wäßr. Ammoniak gewaschen, über $\mathrm{CaCl}_{2}$ getrocknet, das Lösungsmittel abgezogen und der Rückstand destilliert. Nach einem Vorlauf von $0.10 \mathrm{~g}$ nicht umgesetztem $1 \mathrm{~b}$ erhielt man bei $85-105^{\circ} \mathrm{C}(\mathrm{Bad}) / 3$ Torr $1.21 \mathrm{~g}$ $(36 \%)$ eines Gemisches, das neben einer geringen Menge einer nicht identifizierten Substanz 3c und $4 \mathrm{c}$ im Verhältnis 4:1 enthielt.

Isomerisierung von $4 \mathrm{c} z u$ 3c: Ein Teil des oben erhaltenen Gemisches in Methylenchlorid wurde bei $-78^{\circ} \mathrm{C}$ zu $4 \mathrm{ml} \mathrm{Zinn(IV)-chlorid} \mathrm{in} 25 \mathrm{ml} \mathrm{CH}_{2} \mathrm{Cl}_{2}$ getropft, $12 \mathrm{~h}$ bei $-78^{\circ} \mathrm{C}$ gerührt, sodann wie oben aufgearbeitet: reines 1-Chlor-5,5-dimethyl-2-octen-6-in (3c). - IR (Film): 2960, 2910, 2860, $3030(\mathrm{CH}), 975 \mathrm{~cm}^{-1}$ (CH-wagging, trans-Olefin). - ${ }^{1} \mathrm{H}$-NMR: Tab. 2.

3-Chlor-5,5-dimethyl-1-octen-6-in (4c), im Gemisch mit 3c identifiziert: IR (Film): 2960, 2910, $2860,3030,3070(\mathrm{CH}), 1660,1635(\mathrm{C}=\mathrm{C}), 975 \mathrm{mit}$ Schulter bei ca. $990,930 \mathrm{~cm}^{-1}$ (CH-wagging). - 'H-NMR $\left(\mathrm{CCl}_{4}\right): \delta=1.21\left(\mathrm{~s} ; \mathrm{CH}_{3}\right), 1.25\left(\mathrm{~s} ; \mathrm{CH}_{3}\right), 1.77(\mathrm{~s} ; 8-\mathrm{H}), 1.93(\mathrm{~d}, J=6 \mathrm{~Hz} ; 4-\mathrm{H}), 4.59$ $(\mathrm{mc} ; 3-\mathrm{H}), 5.0\left(\mathrm{dd}, J_{\text {gem }}=2 \mathrm{~Hz}, J_{\text {cis }}=10 \mathrm{~Hz} ; 1-\mathrm{H}\right), 5.20\left(\mathrm{dd}, J_{\text {gem }}=2, J_{\text {trans }}=17 \mathrm{~Hz} ; 1-\mathrm{H}\right)$, 5.93 (von $3 \mathrm{c}$ überlagertes ddd; $2-\mathrm{H}$ ).

Cyclisierung von 3c: Unter $\mathrm{N}_{2}$-Schutz wurden $1.00 \mathrm{~g}$ eines Gemisches aus $3 \mathrm{c}$ und $4 \mathrm{c}$ sowie $11 \mathrm{~g}$ Zinkchlorid in $13 \mathrm{ml}$ Ether und $130 \mathrm{ml} \mathrm{CH}_{2} \mathrm{Cl}_{2} 48 \mathrm{~h}$ bei $0^{\circ} \mathrm{C}$ gerührt. Nach der üblichen Aufarbeitung mit eiskaltem konz. wäßr. Ammoniak, Trocknen und Abziehen des Lösungsmittels wurde unter Hydrochinonzusatz destilliert. Nach einer Vorfraktion von Cymol (nicht eindeutig identifi-

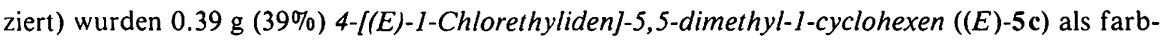
loses Öl mit Sdp. $65-80^{\circ} \mathrm{C}(\mathrm{Bad}) / 3$ Torr erhalten. - ${ }^{1} \mathrm{H}-\mathrm{NMR}$ : Tab. 3.

$$
\mathrm{C}_{10} \mathrm{H}_{15} \mathrm{Cl}(170.7) \quad \text { Ber. C } 70.37 \mathrm{H} 8.86 \text { Gef. C } 70.15 \mathrm{H} 8.69
$$

4. $1 \mathrm{~b}$ und Isopren (2 b): $2.32 \mathrm{~g} \mathrm{(19.9} \mathrm{mmol)} 1 \mathrm{~b}$ und $1.36 \mathrm{~g} \mathrm{(20.0} \mathrm{mmol)} 2 \mathrm{~b}$ in $10 \mathrm{ml} \mathrm{CH}_{2} \mathrm{Cl}_{2}$ wurden bei $-78^{\circ} \mathrm{C}$ zu einer Lösung von $1.1 \mathrm{~g} \mathrm{ZnCl}_{2}$ in $1.3 \mathrm{ml}$ Ether und $23 \mathrm{ml} \mathrm{CH}_{2} \mathrm{Cl}_{2}$ getropft. Nach $44 \mathrm{~h}$ Rühren wurde wie üblich mit wäßr. Ammoniak aufgearbeitet. Destillation ergab $0.22 \mathrm{~g} \mathbf{~ b}$

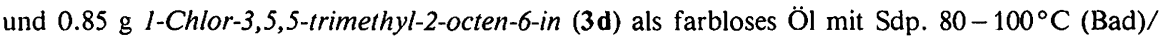
3 Torr. Ausb. 26\%, bezogen auf umgesetztes 1b. - IR $\left(\mathrm{CCl}_{4}\right): 3000,2950,2890(\mathrm{CH}), 1655$ $\mathrm{cm}^{-1}(\mathrm{C}=\mathrm{C}) .-{ }^{1} \mathrm{H}-\mathrm{NMR}$ : Tab. 2. - MS $(70 \mathrm{eV}): m / e=186,184\left(2 \%, 9 \%, \mathrm{M}^{+}\right), 171,169(2$, 5), 149 (46), 148 (42), 133 (100), 107 (37), 105 (53), 91 (52), 81 (44).

$$
\mathrm{C}_{11} \mathrm{H}_{17} \mathrm{Cl} \text { (184.7) Ber. C } 71.53 \mathrm{H} \mathrm{9.28} \text { Gef. C } 71.43 \mathrm{H} 9.60
$$

Cyclisierung von 3d: Die Lösung von $1.20 \mathrm{~g} \mathrm{3d}$ in $20 \mathrm{ml} \mathrm{CH}_{2} \mathrm{Cl}_{2}$ wurde innerhalb von $1.5 \mathrm{~h}$ bei $0^{\circ} \mathrm{C} \mathrm{zu} 2.75 \mathrm{~g} \mathrm{ZnCl}_{2}$ in $3.2 \mathrm{ml}$ Ether und $110 \mathrm{ml} \mathrm{CH}_{2} \mathrm{Cl}_{2}$ getropft. Nach $5 \mathrm{~h}$ wurde wie üblich aufgearbeitet und unter Zusatz von Hydrochinon destilliert: $0.49 \mathrm{~g} \mathrm{4-[(E)-1-Chlorethyliden]-1,5,5-}$ trimethyl-1-cyclohexen $((E)-5 \mathrm{~d})$ als farbloses Öl mit Sdp. $70-85^{\circ} \mathrm{C}(\mathrm{Bad}) / 3 \mathrm{Torr}$. 5d war noch durch eine aromatische Verbindung verunreinigt, die destillativ nicht abgetrennt werden konnte, und wurde daher nur durch das ${ }^{1} \mathrm{H}$-NMR-Spektrum (Tab. 3) identifiziert.

5. $1 \mathrm{~b}$ und 4-Methyl-1,3-pentadien (2f): Eine Lösung von $1.32 \mathrm{~g}(11.3 \mathrm{mmol}) 1 \mathrm{~b}$ und $0.82 \mathrm{~g}$ ( $10.0 \mathrm{mmol}) 2 \mathrm{f}$ in $5 \mathrm{ml} \mathrm{CH} \mathrm{Cl}_{2}$ wurde bei $-78^{\circ} \mathrm{C}$ zu einer Lösung von $1.0 \mathrm{~g} \mathrm{ZnCl}_{2}$ in $1.2 \mathrm{ml} \mathrm{Ether}$ und $23 \mathrm{ml} \mathrm{CH}_{2} \mathrm{Cl}_{2}$ getropft, $15 \mathrm{~h}$ bei $-78^{\circ} \mathrm{C}$ belassen und in üblicher Weise aufgearbeitet. Destillation bei 0.1 Torr bis zu einer Badtemp. von $120^{\circ} \mathrm{C}$ ergab $0.65 \mathrm{~g} \mathbf{~ b}$ sowie $0.99 \mathrm{~g}$ harzigen Rückstand.

6. 3-Chlor-3-methyl-1-phenyl-1-butin (1c) und 1,3-Butadien (2a): Eine Lösung von $4.2 \mathrm{~g} \mathrm{ZnCl}_{2}$ in $5.0 \mathrm{ml}$ Ether und $10 \mathrm{ml} \mathrm{CH} \mathrm{Cl}_{2}$ wurde bei $-78^{\circ} \mathrm{C}$ mit $4.32 \mathrm{~g}(79.9 \mathrm{mmol}) 2 \mathrm{a}$ versetzt; dazu wurde unter Rühren eine Lösung von $3.56 \mathrm{~g}(19.9 \mathrm{mmol}) 1 \mathrm{c}$ in $10 \mathrm{ml} \mathrm{CH}_{2} \mathrm{Cl}_{2}$ getropft und $48 \mathrm{~h}$ bei $-78^{\circ} \mathrm{C}$ gerührt. Das Reaktionsgemisch wurde auf eiskaltes konz. wäßr. Ammoniak gegossen und gründlich durchgeschüttelt, wobei sich die vorher dunkelrote Methylenchlorid-Phase ent-

Chem. Ber. 115 (1982) 
färbte. Die organische Phase wurde abgetrennt, nochmals mit konz. wäßr. Ammoniak gewaschen, über $\mathrm{CaCl}_{2}$ getrocknet, das Lösungsmittel abgezogen und der Rückstand destilliert. Nach einem Vorlauf von $315 \mathrm{mg}(1.8 \mathrm{mmol}) 1 \mathrm{c}$ erhielt man bei $90-110^{\circ} \mathrm{C}(\mathrm{Bad}) / 0.1$ Torr $1.95 \mathrm{~g}(46 \%$, bezogen auf umgesetztes $1 \mathrm{c}$ ) einer farblosen Flüssigkeit, die nach ${ }^{1} \mathrm{H}$-NMR aus $40 \% \mathbf{3 f}, 40 \% \mathbf{4 f}$ und $20 \% 5 \mathrm{f}$ besteht.

Isolierung von 4-[(E)- $\alpha$-Chlorbenzyliden]-5,5-dimethyl-1-cyclohexen $((E)-5 \mathrm{f}): 1.65 \mathrm{~g}$ des Gemisches aus $3 \mathrm{f}, \mathbf{4} f$ und $\mathbf{5 f}$ liefern beim 24stdg. Behandeln mit $2.8 \mathrm{~g} \mathrm{ZnCl}_{2}$ in $3.4 \mathrm{ml}$ Ether und $200 \mathrm{ml}$ $\mathrm{CH}_{2} \mathrm{Cl}_{2}$ bei $25^{\circ} \mathrm{C} 1.10 \mathrm{~g}(67 \%)(E)-5 \mathrm{f}$ als farblose Flüssigkeit mit Sdp. $85-95^{\circ} \mathrm{C}(\mathrm{Bad}) / 0.1 \mathrm{Torr}$. ${ }^{1} \mathrm{H}-\mathrm{NMR}$ : Tab. 3. $5 \mathrm{f}$ war durch eine Spur eines unbekannten Isomeren verunreinigt.

$$
\mathrm{C}_{15} \mathrm{H}_{17} \mathrm{Cl} \text { (232.7) Ber. C } 77.40 \mathrm{H} 7.36 \text { Gef. C } 77.54 \mathrm{H} 7.45
$$

Isolierung von (E)-7-Chlor-3,3-dimethyl-1-phenyl-5-hepten-1-in (3f): $0.20 \mathrm{~g}$ des 2:2:1Gemisches aus $3 \mathrm{f}, \mathbf{4 f}$ und $5 \mathrm{f}$ gehen bei $0^{\circ} \mathrm{C}$ beim Behandeln mit $1.1 \mathrm{~g} \mathrm{ZnCl}_{2}$ in $1.2 \mathrm{ml}$ Ether und $25 \mathrm{ml} \mathrm{CH}_{2} \mathrm{Cl}_{2}$ innerhalb von $15 \mathrm{~min}$ in ein 2:3-Gemisch aus $3 \mathbf{f}$ und $\mathbf{5 f}$ über, das durch SC (Kieselgel, Petrolether) getrennt wurde, wobei $5 \mathrm{f}$ rascher wanderte als 3f. - IR (Film) von 3f: 2970, 2930, 2870, 3030, 3060, $3090(\mathrm{CH}), 2220(\mathrm{C} \equiv \mathrm{C}), 1665(\mathrm{C}=\mathrm{C}), 1490,1600$ (Aromaten-Gerüst), 970 (trans-Alken), 755, 690, $785 \mathrm{~cm}^{-1}$ (CH-wagging). - ${ }^{1} \mathrm{H}$-NMR: Tab. 2.

3-Chlor-5,5-dimethyl-7-phenyl-1-hepten-6-in (4f) konnte nicht in Substanz isoliert werden und wurde im Gemisch mit $3 \mathbf{f}$ und $\mathbf{5 f}$ identifiziert. $-{ }^{1} \mathrm{H}-\mathrm{NMR}\left(\mathrm{CCl}_{4}\right): \delta=1.30\left(\mathrm{~s} ; \mathrm{CH}_{3}\right), 1.36$ (s; $\mathrm{CH}_{3}$ ), 2.02 (d, $J=6 \mathrm{~Hz} ; 4-\mathrm{H}$ ), 4.63 (br. q, $J_{3,4} \approx J_{2,3} \approx 7 \mathrm{~Hz} ; 3-\mathrm{H}$ ), olef. Protonen nicht mit Sicherheit zuzuordnen.

7. $1 \mathrm{c}$ und Isopren (2b): $\mathrm{Zu}$ einer Lösung von $1.1 \mathrm{~g} \mathrm{ZnCl}_{2}$ in $1.3 \mathrm{ml}$ Ether und $23 \mathrm{ml} \mathrm{CH}_{2} \mathrm{Cl}_{2}$ wurden bei $-78^{\circ} \mathrm{C}$ innerhalb von $30 \mathrm{~min} 3.56 \mathrm{~g}(19.9 \mathrm{mmol}) 1 \mathrm{c}$ und $1.36 \mathrm{~g}(20.0 \mathrm{mmol}) 2 \mathrm{~b}$ in $10 \mathrm{ml} \mathrm{C} \mathrm{CH}_{2} \mathrm{Cl}_{2}$ getropft. Nach $1.5 \mathrm{~h}$ Rühren wurden nochmals $0.36 \mathrm{~g}(5.3 \mathrm{mmol}) 2 \mathrm{~b}$ in $5 \mathrm{ml}$ $\mathrm{CH}_{2} \mathrm{Cl}_{2}$ zugetropft und noch weitere $2 \mathrm{~h}$ bei $-78^{\circ} \mathrm{C}$ belassen. Nach der üblichen Aufarbeitung mit konz. wäßr. Ammoniak wurde destilliert. Im Vorlauf wurden $0.45 \mathrm{~g}(2.5 \mathrm{mmol}) 1 \mathrm{c}$ zurückgewonnen. Die Hauptfraktion bei $100-140^{\circ} \mathrm{C}(\mathrm{Bad}) / 0.1$ Torr bestand aus $91 \% 3 \mathrm{~g}$ und $9 \%(E)-5 \mathrm{~g}$. Gesamtausbeute $2.87 \mathrm{~g}(67 \%$, bezogen auf umgesetztes $1 \mathrm{c})$.

7-Chlor-3,3,5-trimethyl-1-phenyl-5-hepten-1-in (3g): Sdp. 105-115 ${ }^{\circ} \mathrm{C}(\mathrm{Bad}) / 0.1$ Torr. - IR (Film): 2960, 2920, 2870, 3050, 3020, $3080(\mathrm{CH}), 2225(\mathrm{C} \equiv \mathrm{C}), 1650(\mathrm{C}=\mathrm{C}), 1490,1595,1570$ (Aromaten-Gerüst), 690, 755, 860, $800 \mathrm{~cm}^{-1}$ (CH-wagging). - ${ }^{1} \mathrm{H}$-NMR: Tab. 2. - MS (70 eV): $m / e=248,246\left(19 \%, 61 \%, \mathrm{M}^{+}\right), 233,231(4,15), 211(100), 195(89), 143(40)$.

$$
\mathrm{C}_{16} \mathrm{H}_{19} \mathrm{Cl}(246.8) \text { Ber. C } 77.87 \mathrm{H} 7.76 \text { Gef. C } 77.41 \mathrm{H} 7.56
$$

Cyclisierung von $3 \mathrm{~g}$ : Die Lösung von $600 \mathrm{mg}$ (2.43 mmol) des oben erhaltenen Gemisches aus $3 \mathrm{~g}$ und $5 \mathrm{~g}$ in $10 \mathrm{ml} \mathrm{CH}_{2} \mathrm{Cl}_{2}$ wurde bei $0^{\circ} \mathrm{C}$ unter Rühren zu einer Lösung von $0.8 \mathrm{~g} \mathrm{ZnCl}_{2}$ in $1 \mathrm{ml}$ Ether und $50 \mathrm{ml} \mathrm{CH}_{2} \mathrm{Cl}_{2}$ getropft. Nach $45 \mathrm{~min}$ wurde das $\mathrm{ZnCl}_{2}$ mit wäßr. Ammoniak ausgewaschen, getrocknet und destilliert: $490 \mathrm{mg}(82 \%) 4-[(E)-\alpha$-Chlorbenzyliden]-1,5,5-trimethyl-1cyclohexen $((E)-5 \mathrm{~g})$ mit Sdp. $100-110^{\circ} \mathrm{C}(\mathrm{Bad}) / 0.1$ Torr. Farblose, hexagonale Platten mit Schmp. $28-30^{\circ} \mathrm{C}$ (Ethanol/Ether), die sich innerhalb von wenigen Tagen bei Raumtemp. zersetzen. - IR (Film): 2950, 2920, 2870, 2820, 3010, $3050(\mathrm{CH}), 1630(\mathrm{C}=\mathrm{C}), 1485,1590$ (AromatenGerüst), 740, $700 \mathrm{~cm}^{-1}$ (CH-wagging). - UV (Cyclohexan): $\lambda_{\max }(\log \varepsilon)=225(3.79), 246.5 \mathrm{~nm}$ (3.59). - ${ }^{1} \mathrm{H}-\mathrm{NMR}$ : Tab. 3. - MS (70 eV): $m / e=248,246\left(11 \%, 39 \%, \mathrm{M}^{+}\right), 244(9), 233,231$ (3, 14), 229 (12), 211 (100), 195 (37).

$$
\mathrm{C}_{16} \mathrm{H}_{19} \mathrm{Cl} \text { (246.8) Ber. C } 77.87 \text { H 7.76 Gef. C } 77.83 \mathrm{H} 7.50
$$

Geschwindigkeit der Cyclisierung von $3 \mathrm{~g}$ : Jeweils $2.4 \mathrm{ml}$ der Lösung von $4.92 \mathrm{~g}(20 \mathrm{mmol})$ eines Gemisches aus $71 \% 3 \mathrm{~g}$ und $29 \% 5 \mathrm{~g}$ in $10 \mathrm{ml} \mathrm{CH}_{2} \mathrm{Cl}_{2}$ wurden bei verschiedenen Temperaturen unter Rühren zu Lösungen aus $0.18 \mathrm{~g}$ Zinkchlorid in $0.21 \mathrm{ml}$ Ether und $3.7 \mathrm{ml} \mathrm{CH}_{2} \mathrm{Cl}_{2}$ getropft. 
Nach 45 min wurden die Reaktionsmischungen auf $20 \mathrm{ml}$ kräftig gerührtes konz. wäßr. Ammoniak (vorgekühlt auf $-10^{\circ} \mathrm{C}$ ) gegossen und 10 min gerührt. Die organische Phase wurde abgetrennt, nochmals mit Ammoniak behandelt, über $\mathrm{CaCl}_{2}$ getrocknet und das Lösungsmittel i. Vak. abgezogen. Durch Integration der ${ }^{1} \mathrm{H}$-NMR-Signale bei $\delta=4.05\left(\mathrm{CH}_{2} \mathrm{Cl}, 3 \mathrm{~g}\right)$ und $3.17\left(3-\mathrm{H}_{2}\right.$, $5 \mathrm{~g}$ ) wurde die Produktzusammensetzung bestimmt.

\begin{tabular}{cccccc}
$\begin{array}{c}\text { Temp. } \\
\left({ }^{\circ} \mathrm{C}\right)\end{array}$ & \multicolumn{2}{c}{ Integration $(\mathbf{c m})$} & $\mathbf{7 0} \mathbf{3 g}$ & $\mathbf{\%} \mathbf{5 g}$ & $\begin{array}{c}\text { \% Cyclisierung in } \\
45 \text { min }\end{array}$ \\
\hline-60 & 4.95 & 1.95 & 71.7 & 28.3 & 0 \\
-50 & 3.70 & 1.80 & 67.3 & 32.7 & 5.7 \\
-40 & 3.35 & 3.00 & 52.8 & 47.2 & 26.0 \\
-30 & 3.00 & 6.00 & 33.3 & 66.7 & 53.3
\end{tabular}

Umsetzung von (E)-5 $\mathrm{g}$ mit Silbertrifluoracetat: Die Lösung von $0.21 \mathrm{~g}(0.85 \mathrm{mmol})(E)-5 \mathrm{~g}$ und $0.50 \mathrm{~g}(2.3 \mathrm{mmol})$ Silbertrifluoracetat in $25 \mathrm{ml}$ Ether wurde $90 \mathrm{~h}$ unter Rückfluß gekocht (Lichtausschluß). Es wurde filtriert, überschüssiges Silbertrifluoracetat mit Wasser ausgewaschen, mit $\mathrm{NaCl}$-Lösung durchgeschüttelt und über $\mathrm{CaCl}_{2}$ getrocknet. Destillation lieferte $0.20 \mathrm{~g} \mathrm{(73 \% )}$ 1,5,5-Trimethyl-4-[(E)- $\alpha$-(trifluoracetoxy)benzyliden]-1-cyclohexen (7) mit Sdp. $85-95^{\circ} \mathrm{C}$ (Bad)/0.05 Torr. - IR (Film): 2990, 2960, 2910, 2850, 3080, $3040(\mathrm{CH}), 1785$ (C=O), 1670 $(\mathrm{C}=\mathrm{C}), 1140,1225,1170\left(\mathrm{CF}_{3}, \mathrm{C}-\mathrm{O}\right), 705,770 \mathrm{~cm}^{-1}$ ( $\mathrm{CH}$-wagging). $-{ }^{1} \mathrm{H}-\mathrm{NMR}\left(\mathrm{CCl}_{4}\right): \delta=$ 0.90 (s; 6H, 5- $\mathrm{CH}_{3}$ ), 1.68 (br. s; 3H, 1- $\mathrm{CH}_{3}$ ), 1.87 (br. s; $\left.2 \mathrm{H}, 6-\mathrm{H}\right), 2.86$ (mc; $2 \mathrm{H}, 3-\mathrm{H}$ ), 5.35 (mc; $1 \mathrm{H}, 2-\mathrm{H}), 7.33\left(\mathrm{~s} ; 5 \mathrm{H}\right.$, Aromaten-H). - MS (70 eV): $m / e=324\left(30 \%, \mathrm{M}^{+}\right), 210(93), 195$ (100). $\quad \mathrm{C}_{18} \mathrm{H}_{19} \mathrm{~F}_{3} \mathrm{O}_{2}(324.3)$ Ber. C $66.65 \mathrm{H} 5.90$ Gef. C $68.95 \mathrm{H} 5.97$

Aminolyse von 7: Die Lösung von $0.20 \mathrm{~g}(0.62 \mathrm{mmol}) 7 \mathrm{in} 30 \mathrm{ml}$ Ether wurde mit $10 \mathrm{ml} \mathrm{konz}$. wäßr. Ammoniak 30 min gründlich durchgeschüttelt. Nach Trennung der Phasen wurde mit Wasser gewaschen, über $\mathrm{CaCl}_{2}$ getrocknet und zur Abtrennung des Trifluoracetamids über wenig Kieselgel filtriert. Destillation lieferte $0.12 \mathrm{~g} \mathrm{(85 \% )}$ Phenyl(4,6,6-trimethyl-3-cyclohexen-1-yl)methanon (8) als farbloses Öl mit Sdp. $80-90^{\circ} \mathrm{C}(\mathrm{Bad}) / 0.1$ Torr, das beim Abkühlen durchkristallisierte. Farblose Spieße mit Schmp. $37-38^{\circ} \mathrm{C}$ (Pentan). - IR (Film): 2950, 2920, $2880(\mathrm{CH}), 1670$ $(\mathrm{C}=\mathrm{O}), 1590,1570$ (Aromaten-Gerüst), $690,725 \mathrm{~cm}^{-1}$ (CH-wagging). $-{ }^{1} \mathrm{H}$-NMR $\left(\mathrm{CCl}_{4}\right): \delta=$ $0.91\left(\mathrm{~s} ; 3 \mathrm{H}, 6-\mathrm{CH}_{3}\right), 1.03\left(\mathrm{~s} ; 3 \mathrm{H}, 6-\mathrm{CH}_{3}\right), 1.66$ (br. s; 3 H, 4- $\left.\mathrm{CH}_{3}\right), 1.85(\mathrm{mc} ; 2 \mathrm{H}, 5-\mathrm{H}), 2.15$ (mc; $2 \mathrm{H}, 2-\mathrm{H}), 3.38(\mathrm{dd}, J=8$ und $6 \mathrm{~Hz} ; 1 \mathrm{H}, 1-\mathrm{H}), 5.34(\mathrm{mc} ; 1 \mathrm{H}, 3-\mathrm{H}), 7.15-7.55(\mathrm{~m} ; 3 \mathrm{H}$, Aromaten-H), $7.8-8.1\left(\mathrm{~m} ; 2 \mathrm{H}\right.$, Aromaten-H). $-\mathrm{MS}(70 \mathrm{eV}): m / e=228\left(22 \%, \mathrm{M}^{+}\right), 213(5)$, 123 (24), 108 (22), 105 (100).

\section{$\mathrm{C}_{16} \mathrm{H}_{20} \mathrm{O}(228.3)$ Ber. C $84.16 \mathrm{H} 8.83$ Gef. C $84.26 \mathrm{H} 8.56$}

8. $1 \mathrm{c}$ und Piperylen (2c): Die Lösung von $3.57 \mathrm{~g}(20.0 \mathrm{mmol}) 1 \mathrm{c}$ und $1.36 \mathrm{~g}(20.0 \mathrm{mmol}) 2 \mathrm{c}$ in $10 \mathrm{ml} \mathrm{CH}_{2} \mathrm{Cl}_{2}$ wurde bei $-78^{\circ} \mathrm{C}$ innerhalb von $45 \mathrm{~min}$ zu einer Lösung von $1.1 \mathrm{~g} \mathrm{ZnCl}_{2}$ in $1.3 \mathrm{ml}$ Ether und $23 \mathrm{ml} \mathrm{CH} \mathrm{Cl}_{2}$ getropft. Nach $4 \mathrm{~h}$ wurde in beschriebener Weise aufgearbeitet. Bei $80-145^{\circ} \mathrm{C}(\mathrm{Bad}) / 0.1$ Torr erhielt man $3.46 \mathrm{~g}(70 \%)$ einer farblosen Flüssigkeit, die nach ${ }^{1} \mathrm{H}$ NMR aus $96 \% 3 \mathrm{~h}$ und $4 \% 5 \mathrm{~h}$ bestand.

(E)-7-Chlor-3,3-dimethyl-1-phenyl-5-octen-1-in (3h): IR (Film): $2235(C \equiv C), 750,685,965$ $\mathrm{cm}^{-1}$ (CH-wagging). - ${ }^{1} \mathrm{H}$-NMR: Tab. 2.

$$
\mathrm{C}_{16} \mathrm{H}_{19} \mathrm{Cl}(246.8) \text { Ber. C } 77.87 \text { H } 7.76 \text { Gef. C } 78.27 \mathrm{H} 7.54
$$

Cyclisierung von $3 \mathrm{~h}$ : Die Lösung von $1.82 \mathrm{~g} 3 \mathrm{~h}$ in $30 \mathrm{ml}$ Methylenchlorid wurde bei $-10^{\circ} \mathrm{C}$ innerhalb von $1 \mathrm{~h}$ unter Rühren zu $2.2 \mathrm{~g} \mathrm{ZnCl}_{2}$ in $2.6 \mathrm{ml}$ Ether und $150 \mathrm{ml} \mathrm{CH}_{2} \mathrm{Cl}_{2}$ getropft. Nach

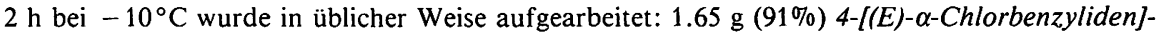
3,5,5-trimethyl-1-cyclohexen $((E)-5 \mathrm{~h})$ als farbloses Öl mit Sdp. $95-105^{\circ} \mathrm{C}(\mathrm{Bad}) / 0.1 \mathrm{Torr}$. - IR (Film): 2960, 2920, 2870, 3020, 2820 (CH), 1490, 1590 (Aromaten-Gerüst), 705, 760, 730, 870

Chem. Ber. 115 (1982) 
$\mathrm{cm}^{-1}$ (CH-wagging). - UV (Cyclohexan): $\lambda_{\max }(\log \varepsilon)=226(3.67), 244 \mathrm{~nm}(3.56) .-{ }^{1} \mathrm{H}-\mathrm{NMR}$ : Tab. 3. - MS (70 eV): $m / e=248,246\left(15 \%, 46 \%, \mathrm{M}^{+}\right), 233$ (2), 231 (6), 211 (58), $205(20), 203$ (61), 195 (29), 169 (100).

$$
\mathrm{C}_{16} \mathrm{H}_{19} \mathrm{Cl}(246.8) \quad \text { Ber. C } 77.87 \text { H } 7.76 \text { Gef. C } 77.84 \mathrm{H} 8.11
$$

9. $1 \mathrm{c}$ und 2,3-Dimethyl-1,3-butadien (2d): Eine Lösung von $3.57 \mathrm{~g} \mathrm{(20.0} \mathrm{mmol)} 1 \mathrm{c}$ und $1.64 \mathrm{~g}$ (20.0 mmol) $2 \mathrm{~d}$ in $10 \mathrm{ml} \mathrm{CH} \mathrm{Cl}_{2}$ tropfte man bei $-78^{\circ} \mathrm{C}$ zu einer Lösung von $2.8 \mathrm{~g} \mathrm{ZnCl}_{2}$ in $3.3 \mathrm{ml}$ Ether und $44 \mathrm{ml} \mathrm{CH} \mathrm{Cl}_{2}$. Nach $3 \mathrm{~h}$ bei $-78^{\circ} \mathrm{C}$ wurden nochmals $0.50 \mathrm{~g}(6.1 \mathrm{mmol}) 2 \mathrm{~d}$ in $5 \mathrm{ml} \mathrm{CH} \mathrm{Cl}_{2}$ zugetropft, weitere $3 \mathrm{~h}$ bei $-78^{\circ} \mathrm{C}$ belassen und dann aufgearbeitet. Destillation lieferte $0.74 \mathrm{~g}(4.1 \mathrm{mmol}) 1 \mathrm{c}$ bei $60-80^{\circ} \mathrm{C}(\mathrm{Bad}) / 0.1$ Torr und $2.45 \mathrm{~g}(59 \%$, bezogen auf umgesetztes 1c) eines 7:3-Gemisches aus (E)- und/oder (Z)-7-Chlor-3,3,5,6-tetramethyl-1-phenyl-5hepten-1-in (3i) und 5i mit Sdp. $105-145^{\circ} \mathrm{C}(\mathrm{Bad}) / 0.1$ Torr. - ${ }^{1} \mathrm{H}-\mathrm{NMR}$ von 3i: Tab. 2.

$$
\mathrm{C}_{17} \mathrm{H}_{21} \mathrm{Cl}(260.8) \quad \text { Ber. C } 78.29 \mathrm{H} 8.12 \text { Gef. C } 78.13 \mathrm{H} 7.96
$$

Cyclisierung von 3i: $1.72 \mathrm{~g}$ des Gemisches aus $3 \mathrm{i}$ und $5 \mathbf{i}$ lieferten innerhalb von $2 \mathrm{~h}$ bei $-10^{\circ} \mathrm{C}$ in Gegenwart von $3.3 \mathrm{~g} \mathrm{ZnCl}_{2}$ und $4 \mathrm{ml}$ Ether in $190 \mathrm{ml} \mathrm{CH}_{2} \mathrm{Cl}_{2} 1.43 \mathrm{~g} \mathrm{(83 \% )} 4-[(E)$ - $\alpha$-Chlorbenzyliden]-1,2,5,5-tetramethyl-I-cyclohexen $((E)-5 \mathrm{i}) \mathrm{mit}$ Sdp. $110-120^{\circ} \mathrm{C}(\mathrm{Bad}) / 0.1 \mathrm{Torr}$. Gut ausgebildete farblose Prismen mit Schmp. $49-50^{\circ} \mathrm{C}$ (Ethanol/Ether). - IR (KBr): 2960, 2860, $3040(\mathrm{CH}), 1630(\mathrm{C}=\mathrm{C}), 699,751,725 \mathrm{~cm}^{-1}$ (CH-wagging). - UV (Cyclohexan): $\lambda_{\max }(\log \varepsilon)=$ 224 (3.85), $247.5 \mathrm{~nm}$ (3.50). - 'H-NMR: Tab. 3. - MS (70 eV): $m / e=262,260(25 \%, 75 \%$, $\left.\mathrm{M}^{+}\right), 247,245$ (5, 16), 225 (91), 224 (38), 219 (13), 217 (41), 209 (55), 184 (48), 183 (51), 143 (49), 135 (56), 122 (73), 94 (100), 91 (86).

\section{$\mathrm{C}_{17} \mathrm{H}_{21} \mathrm{Cl}(260.8)$ Ber. C $78.29 \mathrm{H} 8.12$ Gef. C $78.24 \mathrm{H} 8.30$}

10. $1 \mathrm{c}$ und 1,2-Dimethylencyclohexan (2e): Aus $3.56 \mathrm{~g}(19.9 \mathrm{mmol}) 1 \mathrm{c}, 2.16 \mathrm{~g}(20.0 \mathrm{mmol}) 2 \mathrm{e}$ und $1.1 \mathrm{~g} \mathrm{ZnCl}_{2}$ in $1.3 \mathrm{ml}$ Ether und $34 \mathrm{ml} \mathrm{CH}_{2} \mathrm{Cl}_{2}$ erhielt man nach der üblichen Arbeitsweise innerhalb von $2 \mathrm{~h}$ bei $-40^{\circ} \mathrm{C}$ rohes $5 \mathrm{j}$. Destillation lieferte neben einem Vorlauf von $0.61 \mathrm{~g} \mathrm{(3.4}$ mmol) $1 \mathrm{c} 2.10 \mathrm{~g}(44 \%$, bezogen auf umgesetztes 1c) 3-[(E)- $\alpha$-Chlorbenzyliden]-1,2,3,4,5,6,7,8octahydro-2,2-dimethylnaphthalin $((E)-5 \mathbf{j})$ bei einer Badtemp. von $90-180^{\circ} \mathrm{C} / 0.05 \mathrm{Tor}$, das bei Abkühlen durchkristallisierte. Farblose Quader aus Ethanol/Ether mit Schmp. $47-49^{\circ} \mathrm{C}$. - IR $(\mathrm{KBr}): 2920,2860,2830,2960,2980,3060(\mathrm{CH}), 1630,1640(\mathrm{C}=\mathrm{C}), 1485,1595$ (AromatenGerüst), 745, 700, $720 \mathrm{~cm}^{-1}$ (CH-wagging). - UV (Cyclohexan): $\lambda_{\max }(\log \varepsilon)=224.5(3.62)$, $247.5 \mathrm{~nm}$ (3.32). $-{ }^{1} \mathrm{H}-\mathrm{NMR}$ : Tab. 3. - MS (70 eV): $m / e=288,286\left(33 \%, 100 \%, \mathrm{M}^{+}\right), 273(4)$, 271 (10), 251 (85), 250 (49).

\section{$\mathrm{C}_{19} \mathrm{H}_{23} \mathrm{Cl}(286.8) \quad$ Ber. C $79.56 \mathrm{H} 8.08$ Gef. C $79.80 \mathrm{H} 8.43$}

11. 1 c und 4-Methyl-1,3-pentadien (2f): Die Lösung von $1.64 \mathrm{~g}(20.0 \mathrm{mmol}) 2 \mathrm{f}$ und $1.78 \mathrm{~g}(10.0$ $\mathrm{mmol}) 1 \mathrm{c}$ in $10 \mathrm{ml}$ Methylenchlorid wurde bei $-65^{\circ} \mathrm{C}$ innerhalb von $1 \mathrm{~h}$ unter Rühren zu $1.1 \mathrm{~g}$

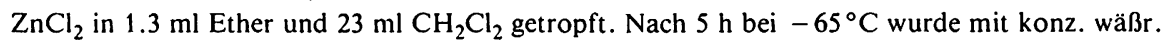
Ammoniak wie üblich aufgearbeitet: $0.80 \mathrm{~g}(23 \%)$ einer farblosen Flüssigkeit bei $155-180^{\circ} \mathrm{C}$ (Bad)/0.05 Torr, die beim Abkühlen erstarte: 7-Chlor-2,2,8,8-tetramethyl-4-(2-methyl-1-propenyl)-9-phenylbicyclo[4.3.0Jnon-9-en (6), farblose Spieße aus Ethanol mit Schmp. 78-80 ${ }^{\circ} \mathrm{C}$. IR (KBr): 2950, 2910, 2850, 3050, $3010(\mathrm{CH}), 1640(\mathrm{C}=\mathrm{C}), 1490,1595$ (Aromaten-Gerüst), 695, $850,735,760,790 \mathrm{~cm}^{-1}$ (CH-wagging). - UV (Cyclohexan): $\lambda_{\max }(\log \varepsilon)=218.5 \mathrm{~nm}(3.67)$. ${ }^{1} \mathrm{H}-\mathrm{NMR}$ : Tab. 4. $-{ }^{13} \mathrm{C}-\mathrm{NMR}\left(\mathrm{CDCl}_{3}\right): \delta=17.9,20.4,25.1,25.7,28.1,30.1\left(6 \mathrm{q} ; 6 \mathrm{CH}_{3}\right), 31.5$ (d; C-4), 35.3, 49.9 (2 s; C-2, -8), 38.3 (t; C-5), 48.7 (t; C-3), 48.8 (d; C-6), 74.7 (d; C-7), 126.5 (d; C-10), 127.3 (d; $C_{\text {meta }}$ ), 129.3, 129.6, 130.2 (3 d; $2 \mathrm{C}_{\text {ortho }}, \mathrm{C}_{\text {para }}$ ), 130.5 (s; C-11), 138.4, 141.5, $141.9\left(3 \mathrm{~s} ; \mathrm{C}_{\text {ipso }}, \mathrm{C}-1,-9\right)$. - MS (70 eV): $m / e=344,342\left(34 \%, 100 \%, \mathrm{M}^{+}\right), 329(14), 327(43)$, 307 (15), 306 (4), 285 (24), 262 (38), 260 (25), 203 (69).

$$
\mathrm{C}_{23} \mathrm{H}_{31} \mathrm{Cl} \text { (342.9) Ber. C } 80.55 \text { H } 9.11 \text { Gef. C } 80.78 \text { H } 9.27
$$


Setzt man 1c und $2 \mathrm{f}$ im Molverhältnis 1:1 um, entsteht ebenfalls 6, und es bleibt eine große Menge an $1 \mathrm{c}$ unumgesetzt zurück.

12. 1c und 2-Methyl-1,3-pentadien (2g) bzw. 2,4-Dimethyl-1,3-pentadien (2h): Eine Lösung von $20.0 \mathrm{mmol} 1 \mathrm{c}$ und $20.0 \mathrm{mmol} 2 \mathrm{~g}$ bzw. $2 \mathrm{~h}$ in $10 \mathrm{ml} \mathrm{CH}_{2} \mathrm{Cl}_{2}$ wurde bei $-78^{\circ} \mathrm{C}$ zu einer Lösung von $1.1 \mathrm{~g} \mathrm{ZnCl}_{2}$ in $1.3 \mathrm{ml}$ Ether und $23 \mathrm{ml} \mathrm{CH}_{2} \mathrm{Cl}_{2}$ getropft. Aufarbeitung nach $6 \mathrm{~h}$ bei $-78^{\circ} \mathrm{C}$ lieferte ein komplexes Produktgemisch, das beim Versuch der Destillation kein Material im für 1:1-Produkte erwarteten Siedepunktsbereich lieferte.

13. 3-Chlor-1,3-diphenyl-1-propin (1 d) und Isopren (2b): $4.53 \mathrm{~g}(20.0 \mathrm{mmol}) \mathbf{1 d}$ und $1.36 \mathrm{~g}$

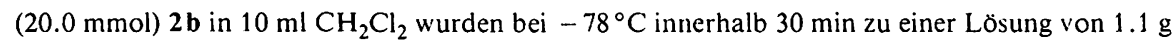

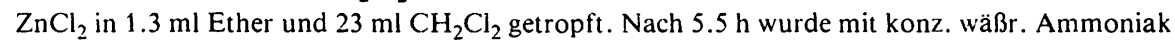
aufgearbeitet. Nach Filtrieren des Rohproduktes über eine kurze Kieselgel-Säule (Petrolether/Cyclohexan 9:1) wurden $4.62 \mathrm{~g} \mathrm{(78 \% )}$ (E)-7-Chlor-5-Inethyl-1,3-diphenyl-5-hepten-1-in (3n) als farbloses Öl erhalten. - IR (Film): 3020, 3050, 2910, $2850(\mathrm{CH}), 1660(\mathrm{C}=\mathrm{C}), 1595$, 1490 (Aromaten-Gerüst), 760, 695, $690 \mathrm{~cm}^{-1}$ (CH-wagging). - 'H-NMR: Tab. 2. - MS (70 eV): $m / e=296,294\left(0.6 \%, 2 \%, \mathrm{M}^{+}\right), 259(8), 258(4), 230$ (7), 228 (8), 191 (21), 149 (100), 133 (98).

Cyclisierung von $3 \mathrm{n}$ : Die Lösung von $3.39 \mathrm{~g}(11.5 \mathrm{mmol}) 3 \mathbf{n}$ in $25 \mathrm{ml} \mathrm{CH}_{2} \mathrm{Cl}_{2}$ wurde bei $0^{\circ} \mathrm{C}$ innerhalb $2.5 \mathrm{~h} \mathrm{zu} 1.1 \mathrm{~g} \mathrm{ZnCl}_{2}$ in $1.3 \mathrm{ml}$ Ether und $80 \mathrm{ml} \mathrm{CH}_{2} \mathrm{Cl}_{2}$ getropft. Es wurde noch $1 \mathrm{~h}$ gerührt und nach der üblichen Aufarbeitung mit konz. wäßr. Ammoniak über eine kurze Säule

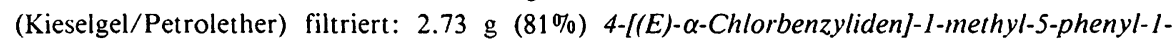
cyclohexen $((E)-\mathbf{5} n)$, das nach Abziehen des Lösungsmittels spontan kristallisierte. Farblose Prismen mit Schmp. 59-61 ${ }^{\circ} \mathrm{C}$ (Ethanol/Ether). - IR (KBr): 2890, 2950, 3000, 2870, 2830, 3040, 3070, $2800(\mathrm{CH}), 1640(\mathrm{C}=\mathrm{C}), 1595,1490$ (Aromaten-Gerüst), 700, 765, $880 \mathrm{~cm}^{-1}$ (CH-wagging). - ${ }^{1} \mathrm{H}-\mathrm{NMR}$ : Tab. 3. $-{ }^{13} \mathrm{C}-\mathrm{NMR}\left(\mathrm{CDCl}_{3}\right): \delta=23.3\left(\mathrm{q}, 1-\mathrm{CH}_{3}\right), 28.6,35.2(2 \mathrm{t}$; C-3, -6), 41.5 (d; C-5), 119.6 (d; C-2), 125.2 (s; CCl), 126.1, 126.7, 128.1, 128.3, 128.9 (5d, C ortho, $\left.\mathrm{C}_{\text {meta }}, \mathrm{C}_{\text {para }}\right), 131.9,135.9\left(2 \mathrm{~s} ; 2 \mathrm{C}_{\text {ipso }}\right), 138.9,142.9(2 \mathrm{~s} ; \mathrm{C}-1,-4) .-\mathrm{MS}(70 \mathrm{eV}): \mathrm{m} / e=296$, $294\left(28 \%, 85 \%, \mathrm{M}^{+}\right), 259(100), 258(35)$.

\section{$\mathrm{C}_{20} \mathrm{H}_{19} \mathrm{Cl}$ (294.8) Ber. C $81.47 \mathrm{H} 6.50$ Gef. C $81.43 \mathrm{H} 6.39$}

14. $1 \mathrm{~d}$ und trans-1,3-Pentadien (2c): Die Lösung von $1.36 \mathrm{~g}(20.0 \mathrm{mmol}) 2 \mathrm{c}$ und $4.53 \mathrm{~g}(20.0$

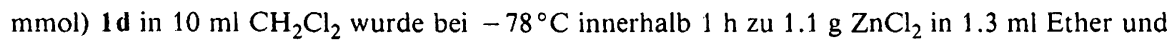
$23 \mathrm{ml} \mathrm{CH} \mathrm{Cl}_{2}$ getropft. Nach $18 \mathrm{~h}$ Rühren bei $-78^{\circ} \mathrm{C}$ wurde wie üblich mit konz. wäßr. Ammoniak aufgearbeitet. Das Rohprodukt bestand nach ${ }^{1} \mathrm{H}-\mathrm{NMR}$ aus 30 und 40 im Verhält nis $2: 1$ und wurde nicht getrennt.

\section{7-Chlor-1,3-diphenyl-5-octen-1-in (3o): 'H-NMR: Tab. 2.}

5-Chlor-1,3-diphenyl-6-octen-1-in (4o), Charakterisierung im Gemisch mit 3o, Signale teilweise verdeckt. $-{ }^{1} \mathrm{H}-\mathrm{NMR}\left(\mathrm{CCl}_{4}\right): \delta=1.66$ (br. d, $\left.J=5 \mathrm{~Hz} ; \mathrm{CH}_{3}\right), 1.9-2.3\left(\mathrm{~m} ; \mathrm{CH}_{2}\right), 3.6-4.1$ (2 m; 5-, 7-H), $5.25-5.85$ (m; olef. H), 7.0-7.5 (Aromaten-H).

Versuch zur Cyclisierung von 30 und 40 bei $-78^{\circ} \mathrm{C}$ : Die Lösung des oben erhaltenen Gemischs aus $3 \mathrm{o}$ und $4 \mathrm{o}(5.70 \mathrm{~g})$ in $25 \mathrm{ml} \mathrm{CH}_{2} \mathrm{Cl}_{2}$ wurde innerhalb $1 \mathrm{~h} \mathrm{zu} 1.1 \mathrm{~g} \mathrm{ZnCl}_{2}$ in $1.3 \mathrm{ml} \mathrm{Ether}$ und $23 \mathrm{ml} \mathrm{CH} \mathrm{Cl}_{2}$ getropft. Nach $42 \mathrm{~h}$ bei $-78^{\circ} \mathrm{C}$ wurde wie üblich mit konz. wäßr. Ammoniak aufgearbeitet. Nach ${ }^{1} \mathrm{H}$-NMR-Spektrum hatte sich die Produktzusammensetzung nicht verändert.

Cyclisierung von 30 und $4 \mathrm{o}: 5.16 \mathrm{~g} \mathrm{(18.0} \mathrm{mmol)} \mathrm{des} \mathrm{Gemisches} \mathrm{aus} 30$ und 40 in $80 \mathrm{ml} \mathrm{CH}_{2} \mathrm{Cl}_{2}$ wurden innerhalb $4.5 \mathrm{~h}$ bei $-10^{\circ} \mathrm{C}$ zu einer Lösung von $2.2 \mathrm{~g} \mathrm{ZnCl}_{2}$ in $2.6 \mathrm{ml}$ Ether und $100 \mathrm{ml}$ $\mathrm{CH}_{2} \mathrm{Cl}_{2}$ getropft. Es wurde noch $2 \mathrm{~h}$ bei $-10^{\circ} \mathrm{C}$ gerührt. Das nach Aufarbeitung erhaltene Isomerengemisch konnte durch SC (Kieselgel/Petrolether) partiell getrennt werden.

Fraktion 1: $1.32 \mathrm{~g} \mathrm{Gemisch}(E)-5 \mathrm{o}-t:(E)-5 \mathrm{o}-c:(Z)-5 \mathrm{o}-c=7: 56: 37$. Aus dieser Fraktion wurden die 'H-NMR-Spektren von 4-[(E)- $\alpha$-Chlorbenzyliden]-r-3-methyl-c-5-phenyl-1-cyclohexen 
((E)-5o-c) und 4-[(Z)- $\alpha$-Chlorbenzyliden]-r-3-methyl-c-5-phenyl-1-cyclohexen ((Z)-50-c) ermittelt (Tab. 3). - MS des Gemisches (70 eV): $m / e=296,294\left(27 \%, 80 \%, \mathrm{M}^{+}\right), 281,279(9,27), 259$ (92), 258 (27), 243 (20), 203 (84), 191 (36), 169 (44), 167 (55), 165 (62), 156 (46), 91 (100).

$$
\mathrm{C}_{20} \mathrm{H}_{19} \mathrm{Cl}(294.8) \text { Ber. C } 81.47 \mathrm{H} 6.50 \text { Gef. C } 81.25 \mathrm{H} 6.53
$$

Fraktion 2: $1.56 \mathrm{~g}$ Gemisch $(E)-50-t:(E)-50-c:(Z)-50-c=75: 15: 10$

Fraktion 3: $1.06 \mathrm{~g}$ 4-[(E)- $\alpha$-Chlorbenzyliden]-r-3-methyl-t-5-phenyl-1-cyclohexen $((E)-50-t)$ als farbloses Öl, das im Tiefkühlfach kristallisierte. - 'H-NMR: Tab. 3. - MS $(70 \mathrm{eV}): m / e=296$, $294\left(32 \%, 95 \%, \mathrm{M}^{+}\right), 281,279(2,7), 259$ (100), 258 (42), 243 (26), 203 (84), 191 (50), 169 (67), 167 (56), 165 (70), $156(59), 91$ (83).

$$
\mathrm{C}_{20} \mathrm{H}_{19} \mathrm{Cl}(294.8) \quad \text { Ber. } \mathrm{C} 81.47 \mathrm{H} 6.50 \text { Gef. C } 82.17 \mathrm{H} 6.21
$$

Fraktion 4: $0.79 \mathrm{~g}$ 4-[(Z)- $\alpha$-Chlorbenzyliden]-r-3-methyl-t-5-phenyl-1-cyclohexen $((Z)-5 \mathrm{o}-t))$ : Farblose Prismen mit Schmp. $102-103^{\circ} \mathrm{C}$ (Ethanol). - UV (Cyclohexan): $\lambda_{\max }(\log \varepsilon)=224.9$ (3.70), $252 \mathrm{~nm}$ (3.60). - ${ }^{1} \mathrm{H}-\mathrm{NMR}$ : Tab. 3. - MS (70 eV): $m / e=296,294\left(31 \%, 94 \%, \mathrm{M}^{+}\right)$, 281, 279 (1, 5), 259 (91), 258 (27), 243 (17), 203 (80), 191 (33), 169 (66), 167 (48), 165 (53), 156 (49), 91 (100).

$$
\mathrm{C}_{20} \mathrm{H}_{19} \mathrm{Cl}(294.8) \quad \text { Ber. C } 81.47 \mathrm{H} 6.50 \text { Gef. C } 81.87 \mathrm{H} 6.42
$$

15. 1d und 2-Methyl-1,3-pentadien (2g): Die Lösung von $2.27 \mathrm{~g}(10.0 \mathrm{mmol}) 1 \mathrm{~d}$ und $0.82 \mathrm{~g}$ ( $10.0 \mathrm{mmol}) 2 \mathrm{~g}$ in $5 \mathrm{ml} \mathrm{CH} \mathrm{Cl}_{2}$ wurde bei $-78^{\circ} \mathrm{C}$ innerhalb $1.5 \mathrm{~h}$ unter Rühren zu einer Lösung von $0.55 \mathrm{~g} \mathrm{ZnCl}_{2}$ in $0.65 \mathrm{ml}$ Ether und $12 \mathrm{ml} \mathrm{CH}_{2} \mathrm{Cl}_{2}$ getropft. Nach $4.5 \mathrm{~h}$ wurde mit konz. wäßr. Ammoniak aufgearbeitet und das Rohprodukt durch Filtrieren über eine Säule (Kieselgel/Petrolether) von Polymeren befreit: Man erhielt $1.77 \mathrm{~g}$ (57\%) 4-( $\alpha$-Chlorbenzyliden)-1,3dimethyl-5-phenyl-1-cyclohexen (5p) als Gemisch von 4 Diastereomeren. - IR (Film): 2970, 2930, 3030, 2880, 3060, $2860(\mathrm{CH}), 1640,1630(\mathrm{C}=\mathrm{C}), 1495,1600$ (Aromaten-Gerüst), 705, 750, $770,720 \mathrm{~cm}^{-1}$ (CH-wagging). - MS $(70 \mathrm{eV}): m / e=310,308\left(3 \%, 10 \%, \mathrm{M}^{+}\right), 273(18), 115$ (36), 105 (40), 91 (100).

$$
\mathrm{C}_{21} \mathrm{H}_{21} \mathrm{Cl} \text { (308.8) Ber. C } 81.67 \mathrm{H} 6.85 \text { Gef. C } 82.40 \mathrm{H} 7.31
$$

Partielle Auftrennung des Isomerengemisches durch SC (Kieselgel, Petrolether) ermöglichte eine Zuordnung der NMR-Signale zu den einzelnen Diastereomeren (Tab. 3).

1) H. Mayr und I. K. Halberstadt-Kausch, Chem. Ber. 115, 3479 (1982).

2) H. Mayr, F. Schütz und I. K. Halberstadt-Kausch, Chem. Ber. 115, 3516 (1982), vorstehend.

3) Vorläufige Mitteilung: H. Mayr und H. Klein, J. Org. Chem. 46, 4097 (1981).

4) Wir danken Herrn Prof. Dr. E. Vilsmaier, Universität Kaiserslautern, für die Aufnahme dieses Spektrums und die Entkopplungsexperimente.

5) H. Mayr und R. Schneider, Chem. Ber. 115, 3470 (1982).

6) I. Fleming, Frontier Orbitals and Organic Chemical Reactions, Wiley, London 1976.

7) H. Mayr, W. Förner und P. v. R. Schleyer, J. Am. Chem. Soc. 101, 6032 (1979); 102, 3663 (1980).

8) H. Mayr, R. Schneider, D. Wilhelm und P. v. R. Schleyer, J. Org. Chem. 46, 5336 (1981).

9) W. D. Pfeifer, C. A. Bahn, P. v. R. Schleyer, S. Bocher, C. E. Harding, K. Hummel, M. Hanack und P. J. Stang, J. Am. Chem. Soc. 93, 1513 (1971).

10) Zur Frage einer konzertierten Cycloaddition von 10c mit $s$-cis-Dienen vgl. Lit. 1).

11) H. Mayr, Angew. Chem. 93, 202 (1981); Angew. Chem., Int. Ed. Engl. 20, 184 (1981).

12) A. Burawoy und E. Spinner, J. Chem. Soc. 1954, 3752.

13) E. Grunwald und S. Winstein, J. Am. Chem. Soc. 70, 846 (1948).

14) A. Streitwieser jr., Solvolytic Displacement Reactions, S. 78, McGraw Hill, New York 1972.

15) R. H. Griffin und J. G. Jewett, J. Am. Chem. Soc. 92, 1104 (1970).

16) E. F. Kiefer und J. D. Roberts, J. Am. Chem. Soc. 84, 784 (1962).

17) M. D. Schiavelli, T. C. Germroth und J. W. Stubbs, J. Org. Chem. 41, 681 (1976).

18) Intermolekulare $[3+2]$-Cycloadditionen von Allyl-Kationen mit Alkinen: $A$. Miller und M. Moore, Tetrahedron Lett. 1980, 577. 